\title{
The Stokes boundary layer for a thixotropic or antithixotropic fluid
}

\author{
by
}

\author{
Catriona R. McArdle \\ David Pritchard \\ Stephen K. Wilson
}





\title{
The Stokes boundary layer for a thixotropic or antithixotropic fluid
}

\author{
Catriona R. McArdle ${ }^{a}$, David Pritchard ${ }^{\mathrm{a}, *}$, Stephen K. Wilson ${ }^{\mathrm{a}, \mathrm{b}}$ \\ ${ }^{a}$ Department of Mathematics and Statistics, University of Strathclyde, 26 Richmond St, \\ Glasgow G1 1XH, Scotland. \\ ${ }^{b}$ Visiting Fellow, Oxford Centre for Collaborative Applied Mathematics (OCCAM), \\ University of Oxford, Mathematical Institute, 24-29 St. Giles', Oxford OX1 3LB.
}

\begin{abstract}
We present a mathematical investigation of the oscillatory boundary layer ('Stokes layer') in a semi-infinite fluid bounded by an oscillating wall (the socalled 'Stokes problem'), when the fluid has a thixotropic or antithixotropic rheology. We obtain asymptotic solutions in the limit of small-amplitude oscillations, and we use numerical integration to validate the asymptotic solutions and to explore the behaviour of the system for larger-amplitude oscillations. The solutions that we obtain differ significantly from the classical solution for a Newtonian fluid. In particular, for antithixotropic fluids the velocity reaches zero at a finite distance from the wall, in contrast to the exponential decay for a thixotropic or a Newtonian fluid.

For small amplitudes of oscillation, three regimes of behaviour are possible: the structure parameter may take values defined instantaneously by the shear rate, or by a long-term average; or it may behave hysteretically. The regime boundaries depend on the precise specification of structure build-up
\end{abstract}

\footnotetext{
${ }^{*}$ Corresponding author. E-mail: david.pritchard@strath.ac.uk. Tel.: $(+44)(0) 141548$
} 3819. Fax: $(+44)(0) 1415483345$. 
and breakdown rates in the rheological model, illustrating the subtleties of complex fluid models in non-rheometric settings. For larger amplitudes of oscillation the dominant behaviour is hysteretic. We discuss in particular the relationship between the shear stress and the shear rate at the oscillating wall.

Keywords: thixotropy, rheopexy, Stokes's second problem, Stokes layer, oscillatory boundary layer

\section{Introduction}

The macroscopic rheological properties of many complex fluids depend on their microscopic structure. This microscopic structure may consist of networks of flocculated colloidal particles, tangles of polymers, or the spatial arrangement of suspended particles or drops [1]. Thixotropic behaviour occurs if, when exposed to high shear rates, the microstructure gradually breaks down and the fluid becomes less viscous, while at low shear rates the microstructure gradually rebuilds and the fluid becomes more viscous. This is common for colloidal dispersions; the converse, antithixotropic (or 'rheopectic') behaviour, is less common but is also known to occur [1]. The time-independent limit of thixotropy is shear-thinning behaviour, while the time-independent limit of antithixotropy is shear-thickening behaviour.

The dynamics of the microstructure may occur on different timescales from the macroscopic flow. When these timescales are comparable with those of the flow, time-dependent rheological models are required to describe the thixotropic or antithixotropic response. Although many such models have been developed over the last fifty years $[1,2]$, much theoretical investiga- 
tion has been restricted to rheometric flows and there is still a lack of generic understanding of how time-dependent rheology manifests itself in other 'classical' fluid-dynamical problems. In this study we consider the effects of thixotropic and antithixotropic behaviour on one such classical flow, the Stokes boundary layer.

Although thixotropy and viscoelasticity are distinct phenomena, a number of recent studies have considered them together, motivated in particular by the extremely complicated rheology of micellar solutions. A model for such fluids was developed by Bautista et al. [3]: this has since been employed both in its full form [4, 5] and in a simplified form [6], while other models have more recently been introduced [7, 8]. As our interest in the present study is in purely viscous structured fluids, such as muds, for which viscoelastic effects are insignificant, we will not discuss viscoelastic models further.

In recent theoretical investigations of thixotropy without elasticity, the most popular structural model has been that introduced by Coussot et al. [9] to describe the 'avalanching' behaviour of mud on slopes. The properties of this model were explored by Roussel et al. [10], and it has subsequently been used to study several related flow problems [11, 12, 13]. A feature of this model is that the build-up rate of the structure is taken to be independent of both the shear and the present state of the structure. In contrast, the structural model used by Billingham \& Ferguson [14] and by Derksen \& Prashant [15], based on the original model by Moore [16], incorporates structure-dependent build-up. With some variations in the choice of constitutive relation, this model has recently been investigated in various geome- 
tries $[17,18,19]$. (Billingham \& Ferguson [14] also incorporated a structure diffusion term, but this has been less widely adopted.) A somewhat more intricate model, with different behaviours during local build-up and breakdown, has recently been developed and applied to flows in rheometers [20]. Most of the rheological models listed above can be represented as special cases of the general model presented by Mewis \& Wagner [2], which we shall describe in section 2.1 .

The present study is concerned with the Stokes problem, sometimes called Stokes's second problem. In the classical version of this problem, a Newtonian fluid of constant kinematic viscosity $\hat{\nu}$ occupies a semi-infinite space $\hat{y}>$ 0 bounded by a rigid impermeable wall at $\hat{y}=0$. This wall is oscillated sinusoidally so that the $\hat{x}$-velocity on the wall is given by $\hat{u}(0, \hat{t})=\hat{u}_{0} \cos (\hat{\omega} \hat{t})$, while the velocity decays to zero as $\hat{y} \rightarrow \infty$. The exact solution is given by

$$
\hat{u}(\hat{y}, \hat{t})=\hat{u}_{0} \exp (-\hat{\gamma} \hat{y}) \cos (\hat{\omega} \hat{t}-\hat{\gamma} \hat{y}), \quad \text { where } \quad \hat{\gamma}=\sqrt{\frac{\hat{\omega}}{2 \hat{\nu}}}
$$

(see e.g. Drazin \& Riley [21], §4.1). This structure is sometimes referred to as an oscillatory boundary layer or 'Stokes layer'. It may be visualised as a sinusoidal travelling wave propagating into the fluid at speed $\hat{\omega} / \hat{\gamma}$ and decaying according to an exponential 'envelope' with characteristic thickness $1 / \hat{\gamma}$, although the dynamics are diffusive rather than wavelike.

The Stokes problem is non-rheometric, because the shear varies in both time and space; it is one of the simplest non-trivial rectilinear flows in which both viscosity and inertia are important. It also provides a useful prototype for more complex flows with oscillatory forcing, such as the boundary layer beneath water waves. Because the oscillation imposes a timescale on the system, the problem becomes particularly interesting when the fluid rheology 
is time-dependent, and its natural timescale may or may not coincide with that of the forcing. This offers opportunities to use the Stokes problem as an unconventional but revealing test for rheological models, as proposed by Balmforth et al. [22].

A few recent studies have investigated the Stokes problem for generalised Newtonian rheologies. Ai \& Vafai [23] tackled the problem numerically for the power-law, Casson and Carreau rheologies, emphasising the transient adjustment from a quiescent to a periodic state. Balmforth et al. [22] conducted a detailed numerical investigation of the Stokes problem for a viscoplastic Herschel-Bulkley fluid, and compared their results with experiments using a kaolin slurry. They suggested that some discrepancies between the numerical and experimental results might be due to thixotropic effects. Most recently, Pritchard et al. [24] revisited the problem for a power-law rheology: they obtained semi-analytical periodic solutions with a non-sinusoidal waveform, and showed numerically that these were attracting even when the wall oscillated sinusoidally. A particularly interesting feature of these solutions was that the boundary layer proved to be of finite thickness for shear-thickening fluids, whereas for shear-thinning fluids the velocity decayed algebraically rather than exponentially with distance from the wall.

Several previous studies have also sought to generalise the classical Stokes problem to viscoelastic fluids. In particular, analytical and approximate solutions have been presented for the second- and third-grade fluid models [25, 26], the Johnson-Segalman model [27] and the Burgers model [28]. Although the decay envelopes and wave propagation speeds in these solutions are somewhat modified from the Newtonian case (1), they do not possess 
qualitatively different features. No systematic tendency was found in these studies for time-dependence to increase or decrease the rate of decay of the 'envelope': indeed, both [25] and [28] found that either could occur depending on the precise model used and the ratio of the elastic relaxation and forcing timescales.

In this study, we employ a combined asymptotic and numerical approach to explore the structure of the Stokes layer for both thixotropic and antithixotropic fluids. We develop asymptotic solutions in the limit of smallamplitude oscillations, which reveal three essentially different ways in which the fluid structure can evolve while satisfying periodicity. We then verify and extend these asymptotic results through direct numerical integration of the governing equations.

In section 2 we describe the rheological model and the governing equations for the problem. We then investigate the two main rheological regimes represented by our model: thixotropic behaviour (section 3) and antithixotropic behaviour (section 4); an intermediate pseudo-Newtonian regime is dealt with in Appendix A. In section 5 we examine 'rheogram' plots of shear stress against shear rate for these solutions, and discuss the information that these plots yield. Finally, in section 6 we summarise our results and comment on the general conclusions that may be drawn from them.

\section{Mathematical model}

\subsection{Rheological model and governing equations}

Mewis \& Wagner's [2] general model for thixotropic and antithixotropic fluids comprises an evolution equation for the scalar structure parameter $\lambda$ 
together with a constitutive relation giving the shear stress tensor $\hat{\tau}_{i j}$ in terms of the shear rate tensor $\hat{e}_{i j}$ and the local value of $\lambda$.

The structure equation is given by

$$
\frac{\mathrm{D} \lambda}{\mathrm{D} \hat{t}}=-\hat{k}_{1} \dot{\gamma}^{a} \lambda^{b}+\hat{k}_{2} \dot{\gamma}^{c}(1-\lambda)^{d}
$$

Here $\hat{k}_{1}$ and $\hat{k}_{2}$ are the rate constants for structural breakdown and build-up respectively and $\dot{\gamma}$ is the total shear rate, while $a, b, c$ and $d$ are non-negative dimensionless exponents. Carets denote dimensional quantities, while dimensionless quantities are unadorned. Note that the structure parameter $\lambda$ is dimensionless and takes values between 0 (a fully unstructured fluid) and 1 (a fully structured fluid).

The four exponents $a, b, c$ and $d$ can, in principle, be chosen independently, so equation (2) can represent a very wide variety of behaviours. We will show in section 2.4 that values $a>c$ correspond to thixotropic behaviour and values $c>a$ to antithixotropic behaviour. This distinction aside, it is assumed by most previous authors that predictions should be relatively insensitive to these choices, so one may choose simple values and concentrate on determining the form of the constitutive equation. (For example, the model of [9] corresponds to $a=1, b=1, c=0, d=0$; that of [14], neglecting the diffusive term, corresponds to $a=1, b=1, c=0, d=1$.) We will show that in the present problem the exponents in (2) determine the regime of behaviour in an unexpectedly subtle manner. This suggests that it may be necessary to pay more attention to the formulation of structure evolution equations than they have tended to receive.

Mewis \& Wagner [2] tabulate a variety of constitutive relations, and there are many possible choices. In the present study, for convenience and to 
allow us to explore the possible regimes of behaviour of equation (2) without introducing an unmanageable number of parameters, we use the simplest of these. This is the relation due to Moore [16] in which the effective viscosity is proportional to $\lambda$,

$$
\hat{\tau}_{i j}=\hat{\eta}_{0} \lambda \hat{e}_{i j}, \quad \text { where } \quad \hat{e}_{i j}=\frac{\partial \hat{u}_{i}}{\partial \hat{x}_{j}}+\frac{\partial \hat{u}_{j}}{\partial \hat{x}_{i}}
$$

and where $\hat{\eta}_{0}$ is a constant viscosity parameter. Note that total shear rate $\dot{\gamma}$ is related to the shear rate tensor $\hat{e}_{i j}$ in the usual manner by

$$
\dot{\gamma}=\sqrt{\frac{1}{2} \sum_{i, j} \hat{e}_{i j} \hat{e}_{i j}}
$$

\subsection{Problem formulation and governing equations}

The flow is governed by the mass conservation and Navier-Stokes equations

$$
\hat{\nabla} \cdot \hat{\mathbf{u}}=0 \quad \text { and } \quad \hat{\rho} \frac{\mathrm{D} \hat{\mathbf{u}}}{\mathrm{D} \hat{t}}=-\hat{\nabla} \hat{p}+\hat{\nabla} \cdot \underline{\hat{\tau}}
$$

where the velocity $\hat{\mathbf{u}}=\hat{u}(\hat{y}, \hat{t}) \mathbf{e}_{x}$, pressure $\hat{p}=\hat{p}(\hat{y}, \hat{t})$ and structure parameter $\lambda=\lambda(\hat{y}, \hat{t})$ are all assumed to be independent of the wall-parallel co-ordinate $\hat{x}$.

From equation (4), the shear rate is given by $\dot{\gamma}=|\partial \hat{u} / \partial \hat{y}|$. The momentum balance in the $\hat{y}$-direction yields $\hat{p}=\hat{p}(\hat{t})$ only so pressure need not be considered, while in the $\hat{x}$-direction combining equation (5) with the constitutive equation (3) yields

$$
\frac{\partial \hat{u}}{\partial \hat{t}}=\hat{\nu}_{0} \frac{\partial}{\partial \hat{y}}\left(\lambda \frac{\partial \hat{u}}{\partial \hat{y}}\right)
$$

where $\hat{\nu}_{0}=\hat{\eta}_{0} / \hat{\rho}$. 
Since $\lambda$ is independent of $\hat{x}$, the convective derivative in the structure equation (2) reduces to $\partial \lambda / \partial \hat{t}$ and hence $\lambda$ satisfies

$$
\frac{\partial \lambda}{\partial \hat{t}}=-\hat{k}_{1}\left|\frac{\partial \hat{u}}{\partial \hat{y}}\right|^{a} \lambda^{b}+\hat{k}_{2}\left|\frac{\partial \hat{u}}{\partial \hat{y}}\right|^{c}(1-\lambda)^{d} .
$$

To solve this system of equations we must specify a boundary condition at the oscillating wall $\hat{y}=0$ and a far-field condition as $\hat{y} \rightarrow \infty$. For simplicity and consistency with the standard Newtonian problem (1) we assume that the wall oscillates sinusoidally with amplitude $\hat{u}_{0}$, so the no-slip condition yields $\hat{u}(0, \hat{t})=\hat{u}_{0} \cos (\hat{\omega} \hat{t})$, and that the velocity decays to zero from the wall so $\hat{u} \rightarrow 0$ as $\hat{y} \rightarrow \infty$.

\subsection{Non-dimensionalisation}

Before proceeding further we non-dimensionalise the problem. We set $\hat{u}=\hat{U}_{0} u, \hat{y}=\hat{Y}_{0} y$ and $\hat{t}=\hat{T}_{0} t$, where the characteristic velocity, length and time scales $\hat{U}_{0}, \hat{Y}_{0}$ and $\hat{T}_{0}$ are given by

$$
\hat{U}_{0}=\hat{\nu}_{0}^{1 / 2}\left(\frac{\hat{k}_{1}^{c-2}}{\hat{k}_{2}^{a-2}}\right)^{1 /(2(a-c))}, \quad \hat{Y}_{0}=\hat{\nu}_{0}^{1 / 2}\left(\frac{\hat{k}_{1}^{c}}{\hat{k}_{2}^{a}}\right)^{1 /(2(a-c))}, \quad \hat{T}_{0}=\left(\frac{\hat{k}_{1}^{c}}{\hat{k}_{2}^{a}}\right)^{1 /(a-c)}
$$

Note that these scales apply only when $a \neq c$. The special case $a=c$, in which the behaviour is 'pseudo-Newtonian', is treated in Appendix A.

The dimensionless problem is therefore given by

$$
\frac{\partial u}{\partial t}=\frac{\partial}{\partial y}\left(\lambda \frac{\partial u}{\partial y}\right)
$$

and

$$
\frac{\partial \lambda}{\partial t}=-\left|\frac{\partial u}{\partial y}\right|^{a} \lambda^{b}+\left|\frac{\partial u}{\partial y}\right|^{c}(1-\lambda)^{d}
$$


subject to the boundary condition $u(0, t)=\epsilon \cos (\omega t)$, where $\epsilon=\hat{u}_{0} / \hat{U}_{0}$ is the non-dimensional amplitude and $\omega=\hat{\omega} \hat{T}_{0}$ the non-dimensionless frequency of the wall oscillations, and subject also to the far-field condition $u \rightarrow 0$ as $y \rightarrow \infty$. Note that since $\hat{T}_{0}$ is defined in terms of the structure response timescales, $\omega$ may be interpreted as a Deborah number for the problem.

When carrying out numerical integrations, we will also specify the initial conditions $u(y, 0)=0$ and $\lambda(y, 0)=$ constant. However, our main interest is in the behaviour at large times. We will show that, as might be expected, transients that depend on the precise initial condition die away and the system adjusts to a periodic state in this limit. When seeking periodic solutions we will impose the periodicity conditions

$$
\lambda(x, t+2 \pi / \omega)=\lambda(x, t) \quad \text { and } \quad u(x, t+2 \pi / \omega)=u(x, t)
$$

for all $x$ and $t$, instead of specifying initial conditions.

Solving equations (9) and (10) is a difficult problem which must, in general, be tackled numerically. However, analytical progress can also be made in the asymptotic limit $\epsilon \rightarrow 0$ corresponding to small amplitude oscillations of the wall. This asymptotic limit is especially informative because it allows us to distinguish different regimes of rheological behaviour, characterised by different responses of the structure to the oscillating shear rate. We will now discuss these possible responses.

\subsection{Instantaneous and time-averaged rheological equilibrium}

It will be useful to distinguish two qualitatively different ways in which the fluid structure may reach an 'equilibrium' with the local shear rate. These 
represent different limiting behaviours of the structure, and will emerge in our asymptotic analysis from different dominant balances in equation (10).

When the response time for the structure is very short compared with the timescale over which the shear rate changes, i.e. in the limit of small Deborah number, $\omega \rightarrow 0$, we may expect the value of $\lambda$ to be instantaneously determined by the shear rate. In this limit, the time derivative in (10) is negligible, and $\lambda=\lambda_{\text {eq }}$, where

$$
\frac{\lambda_{\mathrm{eq}}^{b}}{\left(1-\lambda_{\mathrm{eq}}\right)^{d}}=\left|\frac{\partial u}{\partial y}\right|^{c-a} .
$$

We shall refer to this as 'fast-adjusting' behaviour. The resulting equilibrium stress-shear curve allows us to distinguish between the rheological regimes represented by our model. Since the left-hand side of (12) is a monotonically increasing function of $\lambda_{\text {eq }}$, we see that $\lambda_{\text {eq }}$ increases with increasing shear rate for $a<c$, giving shear-thickening (antithixotropic) behaviour, while $\lambda_{\text {eq }}$ decreases with increasing shear rate for $a>c$, giving shear-thinning (thixotropic) behaviour.

In antithixotropic cases, $a<c$, it is simple to show that the equilibrium shear stress, $\tau_{\text {eq }}=\lambda \partial u / \partial y$, is an increasing function of $\partial u / \partial y$. In thixotropic cases, $a>c$, it is less simple: $\tau_{\text {eq }}$ is an increasing function of $\partial u / \partial y$ for small shear rates, but at large shear rates we obtain the asymptotic result $\left|\tau_{\text {eq }}\right| \sim|\partial u / \partial y|^{(b+c-a) / b}$. Thus, if the shear-thinning effect is strong enough at large shear rates, i.e. if $b+c-a<0$, the model allows $\tau_{\text {eq }}$ to decrease with increasing shear. Since this appears physically unrealistic, we will not consider this parameter range in our study.

When the response time for the structure is very long compared with the timescale over which the shear rate changes, i.e. in the limit of large Deborah 
number, $\omega \rightarrow \infty$, the response is more subtle. Over a period of oscillation the local value of $\lambda$ must remain almost constant and there is no reason in general for it to be in equilibrium with the local shear rate. However, if periodicity is required then there must be rheological equilibrium in a period-averaged sense. Integrating (10) and imposing periodicity gives

$$
0=-\int_{0}^{2 \pi / \omega}\left|\frac{\partial u}{\partial y}\right|^{a} \lambda^{b} \mathrm{~d} t+\int_{0}^{2 \pi / \omega}\left|\frac{\partial u}{\partial y}\right|^{c}(1-\lambda)^{d} \mathrm{~d} t
$$

and since in this limit $\lambda$ is independent of time, we may rearrange equation (13) to yield $\lambda=\lambda_{\mathrm{av}}$, where

$$
\frac{\lambda_{\mathrm{av}}^{b}}{\left(1-\lambda_{\mathrm{av}}\right)^{d}}=\frac{\int_{0}^{2 \pi / \omega}\left|\frac{\partial u}{\partial y}\right|^{c} \mathrm{~d} t}{\int_{0}^{2 \pi / \omega}\left|\frac{\partial u}{\partial y}\right|^{a} \mathrm{~d} t} .
$$

We shall refer to this as 'slow-adjusting' behaviour. Again, the criteria $a \gtrless c$ correspond to thixotropic and antithixotropic behaviour, but now defined in terms of averages of the shear rate of the fluid.

\section{Thixotropic behaviour, $a>c$}

We first consider the case in which the rheology is thixotropic, $a>c$. We will first develop asymptotic solutions in the limit of small-amplitude wall oscillations, $\epsilon \rightarrow 0$, and then extend these numerically.

\subsection{Asymptotic solutions for small-amplitude wall oscillations}

In order to make analytical progress we restrict attention to the asymptotic limit of small-amplitude wall oscillations, $\epsilon \rightarrow 0$. For the rates of structural buildup and breakdown to be comparable in magnitude in this 
limit, the fluid must be highly structured, with $\lambda$ close to unity everywhere. We therefore seek asymptotic solutions of the form

$$
u(y, t)=\epsilon u_{1}(y, t)+o(\epsilon) \quad \text { and } \quad \lambda(y, t)=1-\epsilon^{h} \lambda_{1}(y, t)+o\left(\epsilon^{h}\right),
$$

where the exponent $h>0$ remains to be determined. The boundary conditions are $u_{1}(0, t)=\cos (\omega t)$ and $u_{1} \rightarrow 0$ as $y \rightarrow 0$. Note that because of the thixotropic rheology we may also expect $\lambda_{1}$ to be largest close to the wall where shear rates are highest, and to tend to zero as $y \rightarrow \infty$.

Substituting the expansion (15) into equation (9) and retaining only leading-order terms yields

$$
\frac{\partial u_{1}}{\partial t}=\frac{\partial^{2} u_{1}}{\partial y^{2}}
$$

Solving (16) subject to the boundary and far-field conditions yields

$$
u(y, t) \sim \epsilon u_{1}(y, t)=\epsilon \mathrm{e}^{-\gamma y} \cos (\gamma y-\omega t) \quad \text { with } \quad \gamma=\sqrt{\frac{\omega}{2}},
$$

so at first order we recover the Newtonian solution (1).

Substituting (15) into equation (10) yields

$$
-\epsilon^{h} \frac{\partial \lambda_{1}}{\partial t}=-\epsilon^{a}\left|\frac{\partial u_{1}}{\partial y}\right|^{a}+\epsilon^{c+h d}\left|\frac{\partial u_{1}}{\partial y}\right|^{c} \lambda_{1}^{d}+\text { higher order terms. }
$$

Integrating (18) over a period and applying the periodicity condition (11) then yields

$$
0=-\epsilon^{a} \int_{0}^{2 \pi / \omega}\left|\frac{\partial u_{1}}{\partial y}\right|^{a} \mathrm{~d} t+\epsilon^{c+h d} \int_{0}^{2 \pi / \omega} \lambda_{1}^{d}\left|\frac{\partial u_{1}}{\partial y}\right|^{c} \mathrm{~d} t+\text { higher order terms. }
$$

Since the two terms in (19) must be of the same order in $\epsilon$, we deduce that $a=c+h d$, i.e.

$$
h=\frac{a-c}{d}>0 .
$$


The dominant balance in equation (18) now depends on the relative sizes of the exponents $a$ and $h$, and so three cases must be considered. When $h>a$, the right-hand side of (18) is dominant and so the leading-order behaviour must be an instantaneous balance between the build-up and breakdown terms. This is the 'fast-adjusting' behaviour described in section 2.4, and to leading order the structure parameter must be given by $\lambda \sim \lambda_{\text {eq }}(y, t)$, where $\lambda_{\text {eq }}$ is defined by (12). Conversely, when $h<a$, the left-hand side of (18) is dominant and so the leading-order behaviour must be $\partial \lambda_{1} / \partial t=0$. This is the 'slow-adjusting' behaviour also described in section 2.4, and to leading order the structure parameter must be given by $\lambda \sim \lambda_{\mathrm{av}}(y)$, where $\lambda_{\mathrm{av}}$ is defined by (14). Between these two regimes of behaviour is a marginal case, $h=a$, in which all three terms in (18) are of comparable magnitude and we may expect the behaviour to be intermediate between fast- and slowadjusting behaviour. We shall consider each case in turn.

\subsubsection{Fast-adjusting behaviour when $h>a$}

The first case we consider is $h>a$, when, as we have seen, the structure parameter adjusts rapidly to the local conditions. In this case, $\lambda_{1}$ may be obtained from (18) as

$$
\lambda_{1}(y, t)=\left|\frac{\partial u_{1}}{\partial y}\right|^{h}
$$

and thus

$$
\lambda(y, t) \sim 1-\epsilon^{h} \lambda_{1}=1-\epsilon^{h} \omega^{h / 2}\left|\cos \left(\gamma y-\omega t-\frac{\pi}{4}\right)\right|^{h} \mathrm{e}^{-\gamma h y} .
$$

As expected, the structure parameter approaches unity far from the wall but deviates from unity near the wall. Specifically, the first order deviation of $\lambda$ from unity in (22) is proportional to $\mathrm{e}^{-\gamma h y}$, and so the characteristic width 
of the boundary layer is $1 /(\gamma h)$. Within this exponential envelope, there is an oscillatory variation reflecting the wave-like behaviour of the underlying velocity field.

Also of interest is the shear stress at the wall, $\tau_{\mathrm{w}}$, given by

$$
\tau_{\mathrm{w}}=\left.\lambda \frac{\partial u}{\partial y}\right|_{y=0} \sim-\epsilon \sqrt{\omega} \cos \left(\omega t+\frac{\pi}{4}\right) .
$$

At first order this is identical to the result for a Newtonian fluid.

\subsubsection{Slow-adjusting behaviour when $h<a$}

The second case we consider is $h<a$, when, as we have seen, the structure parameter adjusts slowly to the local conditions. In this case, $\lambda_{1}$ may be obtained from (19) to give

$$
\lambda \sim 1-\epsilon^{h} \lambda_{1}(y)=1-\epsilon^{h} \omega^{h / 2}\left[\frac{\Gamma\left(\frac{a}{2}+\frac{1}{2}\right) \Gamma\left(\frac{c}{2}+1\right)}{\Gamma\left(\frac{a}{2}+1\right) \Gamma\left(\frac{c}{2}+\frac{1}{2}\right)}\right]^{1 / d} \mathrm{e}^{-\gamma h y},
$$

where $\Gamma$ is the usual gamma function. As in the fast-adjusting case, equation (22), the deviation of $\lambda$ from unity decays exponentially with distance from the wall, and the boundary layer has characteristic width $1 /(\gamma h)$. However, in this case there is no oscillation superimposed on the envelope. Also as in the fast-adjusting case, the shear stress at the wall is given to first order by (23).

It is straightforward to demonstrate that if the asymptotic expansion is carried to the next order then the structure parameter must be given by $\lambda \sim 1-\epsilon^{h} \lambda_{1}(y)+\epsilon^{a} \lambda_{2}(y, t)$, where

$$
\frac{\partial \lambda_{2}}{\partial t}=-\left|\frac{\partial u_{1}}{\partial y}\right|^{a}+\left|\frac{\partial u_{1}}{\partial y}\right|^{c} \lambda_{1}^{d}
$$

This result will be of use when we check the validity of these solutions in section 3.1.4. 


\subsubsection{Marginal behaviour when $h=a$}

The third and final case we consider is the marginal case $h=a$, in which all terms in (10) are of comparable magnitude. Now, at leading order (18) becomes

$$
-\frac{\partial \lambda_{1}}{\partial t}=-\left|\frac{\partial u_{1}}{\partial y}\right|^{a}+\left|\frac{\partial u_{1}}{\partial y}\right|^{c} \lambda_{1}^{d}
$$

where $u_{1}(y, t)$ is as before given by $(17)$. Physically, we may expect this case to display behaviour intermediate between fast- and slow- adjusting behaviour.

We seek a solution to (26) that reflects the wavelike structure of the velocity field. Employing the ansatz $\lambda_{1}=f(y) l(\gamma y-\omega t)$, where the functions $f$ and $l$ are to be determined, equation (26) becomes

$f \frac{\partial l}{\partial t}=\left(\mathrm{e}^{-\gamma y} \gamma \sqrt{2}\right)^{a}|\cos (\gamma y-\omega t-\pi / 4)|^{a}-f^{d}\left(\mathrm{e}^{-\gamma y} \gamma \sqrt{2}\right)^{c} l^{d}|\cos (\gamma y-\omega t-\pi / 4)|^{c}$.

We separate variables by requiring that the factors involving $f(y)$ cancel; thus

$$
\frac{\mathrm{e}^{-\gamma a y}(\gamma \sqrt{2})^{a}}{f}=f^{d-1} \mathrm{e}^{-\gamma c y}(\gamma \sqrt{2})^{c}=1
$$

and so

$$
f=\omega^{a / 2} \mathrm{e}^{-\gamma a y} .
$$

This reduces equation (27) to a nonlinear ODE for $l$, which may be written in terms of a new variable $\tau=\omega t-\gamma y+\pi / 4$ as

$$
\omega \frac{\mathrm{d} l}{\mathrm{~d} \tau}+l^{d}|\cos (\tau)|^{c}=|\cos (\tau)|^{a} .
$$

Equation (30) can easily be integrated numerically to obtain the waveform $l(\tau)$; periodicity typically becomes established after only a few periods, although the time taken to reach periodicity increases with $\omega$. The asymptotic 
solution of $\lambda$ then takes the form

$$
\lambda(y, t) \sim 1-\epsilon^{h} \lambda_{1}(y, t)=1-\epsilon^{h} \omega^{h / 2} \mathrm{e}^{-\gamma h y} l(\omega t-\gamma y) .
$$

Note that, once again, the deviation of $\lambda$ from unity is contained within an exponential envelope representing a boundary layer with characteristic width $1 /(\gamma h)=1 /(\gamma a)$, and that once again the shear stress at the wall is given to first order by (23).

The solutions to (30) are interesting because they illustrate how the behaviour in this marginal case mediates between slow- and fast-adjusting as $\omega$ is varied. In the limit $\omega \rightarrow 0$, equation (30) is satisfied by the instantaneous equilibrium

$$
l(\tau)=|\cos (\tau)|^{a}
$$

which corresponds to $\lambda \sim \lambda_{\text {eq }}$. Meanwhile, in the limit $\omega \rightarrow \infty, l$ must become a constant and we recover $\lambda \sim \lambda_{\text {av }}$. Figure 1 illustrates the solutions for $l(\tau)$ for several values of $\omega$. As $\omega$ is increased from zero, the variation of $l(\tau)$ is increasingly attenuated relative to (32), while it also displays an increasing phase lag and an increasingly non-sinusoidal waveform.

\subsubsection{A posteriori estimate of the validity of the asymptotic solutions}

The asymptotic solutions described in the preceding subsections have been obtained in the limit of small-amplitude oscillations, $\epsilon \rightarrow 0$. However, they also depend on the additional independent parameter $\omega$, which represents the dimensionless frequency or Deborah number. It is therefore useful to obtain a posteriori estimates of the validity of these solutions when $\omega$ is not necessarily of order unity. 
It is straightforward to show from (17) that $|\partial u / \partial y|=O\left(\omega^{1 / 2} \epsilon\right)$. In the fast-adjusting regime, (22) yields the scalings $\lambda=O(1), 1-\lambda=O\left(\omega^{h / 2} \epsilon^{h}\right)$ and $\partial \lambda / \partial t=O\left(\omega^{1+h / 2} \epsilon^{h}\right)$. From these, the validity condition for fast adjustment,

$$
\left|\frac{\partial \lambda}{\partial t}\right| \ll\left|\frac{\partial u}{\partial y}\right|^{a} \lambda^{b} \sim\left|\frac{\partial u}{\partial y}\right|^{c}(1-\lambda)^{d}
$$

corresponds to

$$
\epsilon^{h} \omega^{1+h / 2} \ll \epsilon^{a} \omega^{a / 2}, \quad \text { i.e. } \quad \epsilon^{h-a} \omega^{1+(h-a) / 2} \ll 1 .
$$

Fast-adjusting behaviour can therefore be expected in the asymptotic limits of either small oscillations, $\epsilon \rightarrow 0$, or slow oscillations, $\omega \rightarrow 0$.

In the slow-adjusting regime, equation (24) yields the scalings $\lambda=O(1)$ and $1-\lambda=O\left(\omega^{h / 2} \epsilon^{h}\right)$. To estimate the magnitude of $\partial \lambda / \partial t$ we need to consider the next order in $\epsilon$. Integrating equation (25) with respect to $\omega t$ implies that $\omega \lambda_{2}=O\left(\omega^{a / 2}\right)$; thus the validity condition for the asymptotic solution in the slow-adjusting case, namely

$$
\epsilon^{h} \lambda_{1} \gg \epsilon^{a} \lambda_{2}
$$

corresponds to

$$
\epsilon^{h} \omega^{h / 2} \gg \epsilon^{a} \omega^{a / 2-1}, \quad \text { i.e. } \quad \epsilon^{h-a} \omega^{1+(h-a) / 2} \gg 1,
$$

which is exactly the complement of the condition (34) for fast adjustment. Since $h-a<0$ in this regime, this condition always corresponds to small oscillations, $\epsilon \rightarrow 0$, but depending on the relative sizes of $a$ and $h$ it may be obtained when the oscillations are fast or when they are slow.

Figure 2 illustrates the parameter regimes in which the present asymptotic analysis suggests fast-adjusting, slow-adjusting and hysteretic behaviour. To 
avoid ambiguity, we will henceforth refer to the parameter regime $a>c$, $h>a$ as regime $\mathrm{T} 1$ and to the parameter regime $a>c, h<a$ as regime T2; we will reserve the terms 'fast-adjusting' and 'slow-adjusting' for the regimes, depending also on $\epsilon$ and $\omega$, in which the solutions display these behaviours. (The fast-adjusting regime is a subset of $\mathrm{T} 1$ and the slow-adjusting regime is a subset of T2.) When the system is neither fast- nor slow-adjusting, we may expect its behaviour in general to be hysteretic, as shown in figure 2 .

\subsection{Numerical results for finite-amplitude wall oscillations}

We now compare the asymptotic solutions for velocity, structure parameter and shear stress at the wall with numerical solutions obtained using the finite-element package Comsol 3.5a. A finite numerical domain $0 \leq y \leq y_{\max }$ was used, with a domain length of $y_{\max }=10$ unless otherwise stated. The initial conditions were $u(y, 0)=0$ and $\lambda(y, 0)=1$ unless otherwise stated. A zero-velocity condition was applied at $y=y_{\max }$; in general this had a negligible effect on the solution. The mesh comprised 240 equally-spaced grid points (except in the calculations with $\epsilon=10$, where 960 grid points were employed to resolve the behaviour close to the wall), and the numerical relative tolerance was $10^{-6}$. The results reported here were robust to further refinement of these parameters. Run-times ranged from tens of minutes to several hours on a desktop PC.

\subsubsection{Regime T1: $h>a$}

To illustrate the behaviour in regime $\mathrm{T} 1$, in which the structure parameter is fast-adjusting for small $\epsilon$, we take $a=2, b=1, c=1$ and $d=1 / 3$, giving $h=3$. We also set $\omega=1$ so that the condition (34) is easily satisfied. 
We first consider small-amplitude oscillations, $\epsilon=0.01$. Figures 3 a and $3 \mathrm{~b}$ show snapshots of the velocity $u(y, t)$ and structure parameter $\lambda(y, t)$ at equally spaced times during a period. (Note that since the evolution of $\lambda$ is driven by $|\partial u / \partial y|$, it has period $\pi / \omega$ in contrast to the period $2 \pi / \omega$ for $u$; we have therefore plotted $\lambda$ at more frequent intervals than $u$.) The first snapshot is taken at $t=100$, by which time the system has adjusted to an essentially periodic state. The numerical solution (solid lines) and the asymptotic solution (dashed lines) are almost indistinguishable for all values of $y$. As the structure parameter deviates only very weakly from unity (figure $3 \mathrm{~b})$, the velocity field is essentially identical to the Newtonian solution (1), and decays exponentially away from the wall, over a characteristic distance $\gamma^{-1}=\sqrt{2}$. It is clear that the perturbations to the structure parameter decay away from the wall over a shorter distance than the velocity, in accordance with the prediction of equation (22) that they should decay over a characteristic distance $(\gamma h)^{-1}=\sqrt{2} / 3$. There is also evidence in figure $3 \mathrm{~b}$ that the structure parameter $\lambda$ lags slightly behind $\lambda_{\text {eq }}$ at some stages of the oscillation.

To investigate the behaviour for larger oscillations, the amplitude was increased to $\epsilon=1$. Figures $3 \mathrm{c}$ and $3 \mathrm{~d}$ show snapshots of the velocity $u(y, t)$ and structure parameter $\lambda(y, t)$. Some deviation can now be seen from the fast-adjusting asymptotic solution, as the response time of the structure is no longer negligible. The variation of the structure parameter $\lambda$ (figure $3 \mathrm{~d}$ ) is significantly attenuated relative to the instantaneous equilibrium $\lambda_{\text {eq }}$ predicted by the asymptotic solution, and may be described as oscillation around an average equilibrium value. Since $\lambda$ is significantly lower than unity, the 
velocity (figure $3 \mathrm{c}$ ) decays more rapidly with distance from the wall than is predicted by the asymptotic solution (17), in which $\lambda \sim 1$. However, the deviation of the structure parameter from unity is significantly overestimated by the asymptotic solution (22), because shear rates, and thus structure breakdown rates, are generally lower than predicted by the asymptotic solution (17).

When the amplitude is increased further to $\epsilon=10$ (figures 3 e and $\mathrm{f}$ ), these trends are still more strongly pronounced, and the asymptotic description for small $\epsilon$ is clearly no longer valid. The structure has almost completely broken down near the wall (figure $3 \mathrm{f}$ ), and the result of this very low viscosity is that the oscillations in $u$ die out very rapidly (figure $3 \mathrm{e}$ ). The effect of this on the shear stress at the wall will be discussed below.

\subsubsection{Regime T2: $h<a$}

To illustrate the behaviour in regime $\mathrm{T} 2$, in which the structure parameter is slow-adjusting for small $\epsilon$, we take $a=2, b=1, c=1$ and $d=1$, giving $h=1$. We again set $\omega=1$ so that the condition (36) is easily satisfied.

We first consider small-amplitude oscillations, $\epsilon=0.01$. Figure 4 a shows snapshots of the velocity $u(y, t)$ at equally spaced times during a period. The first snapshot is taken at $t=100$, by which time the velocity has adjusted to an essentially periodic state. The numerical solution (solid lines) and the asymptotic solution (dashed lines) are indistinguishable for all values of $y$. As the structure parameter again deviates only very weakly from unity (figure $4 \mathrm{~b}$ ), the velocity field is again essentially identical to the Newtonian solution (1).

Although the velocity adjusts rapidly to an effectively periodic state, the 
structure parameter takes longer to adjust. Figure $4 \mathrm{~b}$ shows plots of $\lambda(y, t)$ at times ranging from $t=100$ to $t=500$. Because the structure parameter varies slowly, it not only remains approximately constant throughout a period but also takes many periods to adjust towards its eventual asymptotic state (the dashed line in figure $4 \mathrm{~b}$ ). The adjustment towards this asymptotic state is fastest close to the wall where shear rates are highest, and is correspondingly slower as shear rates decay exponentially away from the wall.

To investigate the behaviour for larger oscillations, the amplitude was again increased to $\epsilon=1$. Figures $4 \mathrm{c}$ and $\mathrm{d}$ show snapshots of the velocity $u(y, t)$ and structure parameter $\lambda(y, t)$, after the system has adjusted to an essentially periodic state. Some deviation from the asymptotic solution can now be seen. The structure parameter $\lambda$ (figure $4 \mathrm{~d}$ ) is nearly independent of $t$ but strongly dependent on $y$, and oscillates slightly around an average equilibrium value. As in the regime $\mathrm{T} 1$ (figures $3 \mathrm{c}$ and $\mathrm{d}$ ), $\lambda(y, t)$ is significantly lower than unity, so the velocity (figure 4 c) decays more rapidly with distance from the wall than is predicted by the asymptotic solution (17), in which $\lambda \sim 1$. However, the deviation of the structure parameter from unity is again significantly overestimated by the asymptotic solution (24).

When the amplitude is increased further to $\epsilon=10$ (figures 4 e and $\mathrm{f}$ ), the asymptotic description for small $\epsilon$ is clearly no longer valid. Except near the wall, however, the structure parameter still varies only weakly throughout a period (figure $4 \mathrm{f}$ ) so the system still shows essentially slow-adjusting behaviour. What is now conspicuous is that the structure has almost completely broken down near the wall, and the result of this very low viscosity is that the oscillations in $u$ die out very rapidly (figure $4 \mathrm{e}$ ). This is again 
rather similar to the behaviour in regime T1: indeed, with increasing $\epsilon$ the distinction between these regimes becomes increasingly blurred.

\subsubsection{Shear stress at the wall}

It is also of interest to examine the shear stress at the wall, as this is one of the most readily measured quantities in most experimental configurations. Figures 5 a and $5 \mathrm{~b}$ show the shear stress $\tau_{\mathrm{w}}(t)$, normalised by the asymptotic scaling factor $\epsilon$, for regimes $\mathrm{T} 1$ and T2 respectively.

In each case, the variation for small $\epsilon$ is sinusoidal as predicted by the asymptotic solution (23). As $\epsilon$ is increased, the variation becomes slightly less sinusoidal, and the scaling of $\tau_{\mathrm{w}}$ with $\epsilon$ also departs from strict proportionality. There is a competition between the decreasing values of $\lambda$ at the wall and the increasing shear rates, both of which contribute to $\tau_{\mathrm{w}}$. In regime T2 (figure $5 \mathrm{~b}$ ) the former dominates and the rescaled shear stress $\tau_{\mathrm{w}} / \epsilon$ decreases with increasing $\epsilon$. In regime $\mathrm{T} 1$ (figure 5 a) the competition is less straightforward: at first the rescaled shear stress decreases with increasing $\epsilon$, but as $\epsilon$ is increased further the high shear rates near the wall come to dominate and $\tau_{\mathrm{w}} / \epsilon$ increases again. In all these cases, however, the amplitude of $\tau_{\mathrm{w}}$ remains of order $\epsilon$, despite the very strong deviation of both $u$ and $\lambda$ from the asymptotic solution.

When $\epsilon$ is increased to 10, rapid variations of $\tau_{\mathrm{w}}$ occur at times when the shear rate is close to zero (figures $5 \mathrm{a}$ and b). In order to explore this behaviour, in figure 6 we plot $\tau_{\mathrm{w}} / \epsilon$ along with the structure parameter $\lambda$ and the shear rate, also evaluated at the wall. Note that a normalised shear rate $\dot{\gamma}^{*}=(\partial u / \partial y) / \max (\partial u / \partial y)$ has been plotted to allow all three quantities to appear on the same axes. 
In general, the strong oscillation means that $\lambda$ is very low near to the wall. Since $\lambda$ also sets the momentum diffusivity, this means that there is generally a high-shear region near the wall. Throughout most of a period, then, $\lambda$ is low and thus $\partial u / \partial y$ is large; in fact throughout this phase of the oscillation $\lambda$ is gradually decaying. However, because the wall oscillates, there must come a point in the cycle when $\partial u / \partial y$ becomes close to zero. At this point, a feedback process occurs: $\lambda$ increases, thus increasing the momentum diffusivity and further reducing the shear rate. The result of this is that $\lambda$ grows rapidly, while $\partial u / \partial y$ is held close to zero for an extended period (the 'plateau' which can be seen every time the dashed line in figures $5 \mathrm{a}$ and b crosses the axis). Eventually, though, the continued increase of $u$ at the wall causes the shear rate to increase again; increasing shear decreases $\lambda$, which in turn further increases shear. Thus $\lambda$ decreases rapidly until the phase of low $\lambda$ and high shear resumes.

From figures $5 \mathrm{a}$ and $\mathrm{b}$ it is apparent that the rapid variations in $\tau_{\mathrm{w}}$ are the signature of this rather complicated interaction between structure and shear rate. We will see this signature in a different form in section 5.

\subsubsection{Marginal regime $h=a$}

Finally, to illustrate the behaviour in the marginal regime $h=a$ we take $a=2, b=1, c=1$ and $d=1 / 2$, giving $h=a=2$. As in the two previously considered regimes, we set $\omega=1$.

We first consider small-amplitude oscillations with $\epsilon=0.01$. The velocity $u(y, t)$ (figure $7 \mathrm{a}$ ) behaves qualitatively in the same way as in regimes $\mathrm{T} 1$ and T2 (figures 3 a and $4 \mathrm{a}$ ). Although the structure parameter $\lambda(y, t)$ (figure $7 \mathrm{~b}$ ) varies throughout a period, its variation is relatively weak, and qualitatively 
resembles that in regime T2 for larger values of $\epsilon$ (e.g. figure $4 \mathrm{~d})$.

Increasing $\epsilon$ further, first to $\epsilon=1$ (figures $7 \mathrm{c}$ and $\mathrm{d}$ ) and then to $\epsilon=10$ (figures 7 e and f) changes the behaviour quantitatively rather than qualitatively. As in both the previously-considered regimes, the structure breaks down substantially at the wall, with a corresponding very rapid decrease in the amplitude of the velocity variation with distance from the wall.

The shear stress at the wall $\tau_{\mathrm{w}}$ (figure 8 ) is also similar to that in the other two regimes: as in the 'fast-adjusting' regime (figure 5 a) the competition between decreasing structure and increasing shear rates at the wall is rather finely balanced, so that although the variation of $\tau_{\mathrm{w}}(t)$ deviates from the sinusoidal asymptotic solution (23) its amplitude continues to scale roughly with $\epsilon$. Again, when $\epsilon=10$ we see rapid variations as the shear rate approaches zero. The reasons for these are the same as in regimes T1 and T2 (figure 5).

\section{Antithixotropic behaviour, $a<c$}

We now consider the case in which the rheology is antithixotropic, $a<c$. As in the thixotropic case, we will first develop asymptotic solutions in the limit of small-amplitude wall oscillations, $\epsilon \rightarrow 0$, and then extend these numerically.

\subsection{Asymptotic solutions for small-amplitude wall oscillations}

As before, in order to make analytical progress we first restrict attention to the asymptotic limit of small amplitude wall oscillations, $\epsilon \rightarrow 0$. For the rates of structural buildup and breakdown to be comparable in magnitude 
in this limit, the fluid must be highly unstructured, with $\lambda$ close to zero everywhere. We therefore seek asymptotic solutions of the form

$$
u(y, t)=\epsilon u_{1}(y, t)+o(\epsilon) \quad \text { and } \quad \lambda(y, t)=\epsilon^{\beta} \Lambda_{1}(y, t)+o\left(\epsilon^{\beta}\right),
$$

where the exponent $\beta>0$ remains to be determined. The boundary conditions are the same as in the thixotropic case, $u_{1}(0, t)=\cos (\omega t)$ and $u_{1} \rightarrow 0$ as $y \rightarrow 0$. Note that because of the antithixotropic rheology we may also expect $\Lambda_{1}$ to be largest close to the wall where shear rates are highest, and to tend to zero as $y \rightarrow \infty$.

Substituting the expansion (37) into equation (9) and retaining only leading-order terms yields

$$
\frac{\partial u_{1}}{\partial t}=\epsilon^{\beta} \frac{\partial}{\partial y}\left(\Lambda_{1} \frac{\partial u_{1}}{\partial y}\right)
$$

To eliminate $\epsilon$ from this equation we rescale using $y=\epsilon^{\beta / 2} \hat{y}$, where $\hat{y}$ is of order unity, yielding

$$
\frac{\partial u_{1}}{\partial t}=\frac{\partial}{\partial \hat{y}}\left(\Lambda_{1} \frac{\partial u_{1}}{\partial \hat{y}}\right)
$$

Substituting the expansion (37) into equation (10) yields

$\epsilon^{\beta} \frac{\partial \Lambda_{1}}{\partial t}=-\epsilon^{a(1-\beta / 2)+b \beta}\left|\frac{\partial u_{1}}{\partial \hat{y}}\right|^{a} \Lambda_{1}^{b}+\epsilon^{c(1-\beta / 2)}\left|\frac{\partial u_{1}}{\partial \hat{y}}\right|^{c}+$ higher order terms. (40)

Balancing powers of $\epsilon$ on the right hand side yields

$$
\beta=\frac{2(c-a)}{2 b+(c-a)}>0
$$

This simplifies equation (40) to

$$
\epsilon^{\nu} \frac{\partial \Lambda_{1}}{\partial t}=-\left|\frac{\partial u_{1}}{\partial \hat{y}}\right|^{a} \Lambda_{1}^{b}+\left|\frac{\partial u_{1}}{\partial \hat{y}}\right|^{c}+\text { higher order terms, }
$$


where

$$
\nu=\frac{2(c-a-b c)}{2 b+c-a} .
$$

Integrating (40) over a period and applying the periodicity condition (11) then yields

$$
0=-\int_{0}^{2 \pi / \omega} \Lambda_{1}^{b}\left|\frac{\partial u_{1}}{\partial \hat{y}}\right|^{a} \mathrm{~d} t+\int_{0}^{2 \pi / \omega}\left|\frac{\partial u_{1}}{\partial \hat{y}}\right|^{c} \mathrm{~d} t+\text { higher order terms. }
$$

The dominant balance in equation (42) now depends on the sign of the exponent $\nu$, and so three cases must be considered. When $\nu>0$, the righthand side of (42) is dominant and so the leading-order behaviour must be an instantaneous balance between the build-up and breakdown terms. This is the 'fast-adjusting' regime described in section 2.4, and to leading order the structure parameter must be given by $\lambda \sim \lambda_{\text {eq }}(y, t)$, where $\lambda_{\text {eq }}$ is defined by (12). Conversely, when $\nu<0$, the left-hand side of (42) is dominant and so the leading-order behaviour must be $\partial \Lambda_{1} / \partial t=0$. This is the 'slow-adjusting' regime also described in section 2.4, and to leading order the structure parameter must be given by $\lambda \sim \lambda_{\mathrm{av}}(y)$, where $\lambda_{\mathrm{av}}$ is defined by (14). Between these two regimes is a marginal case, $\nu=0$, in which all three terms in (42) are of comparable magnitude and we may expect the behaviour to be intermediate between fast- and slow- adjusting behaviour. We shall consider each case in turn.

\subsubsection{Fast-adjusting behaviour when $\nu>0$}

The first case we consider is $\nu>0$, when, as we have seen, the structure parameter adjusts rapidly to the local conditions. In this case, $\Lambda_{1}$ may be obtained from (42) as

$$
\Lambda_{1}(y, t)=\left|\frac{\partial u_{1}}{\partial \hat{y}}\right|^{(c-a) / b}
$$


and thus from (39)

$$
\frac{\partial u_{1}}{\partial t}=\frac{\partial}{\partial \hat{y}}\left(\left|\frac{\partial u_{1}}{\partial \hat{y}}\right|^{(c-a) / b} \frac{\partial u_{1}}{\partial \hat{y}}\right) .
$$

Equation (46) is exactly the governing equation for a power-law fluid with power-law index $n=(b+c-a) / b>1$ and so the solution for $u_{1}$ is identical to that for a shear-thickening power-law fluid [24].

The most distinctive feature of Stokes flow in a shear-thickening powerlaw fluid is the boundary layer is of finite width. Also, even in the limit $\epsilon \rightarrow 0$, the waveforms of the velocity are non-sinusoidal, unlike those for Newtonian and thixotropic fluids, and so the boundary condition at $y=0$ is not satisfied precisely by the asymptotic solution. The velocity decays algebraically towards the edge of the boundary layer, with exponent $\alpha=$ $(2 b+c-a) /(c-a)$.

Also of interest is the shear stress at the wall, $\tau_{\mathrm{w}}$, given, to first order, by

$$
\tau_{\mathrm{w}} \sim \epsilon^{1+\beta / 2}\left|\frac{\partial u_{1}}{\partial y}\right|^{(c-a) / b} \frac{\partial u_{1}}{\partial y} .
$$

\subsubsection{Slow-adjusting behaviour when $\nu<0$}

The second case we consider is $\nu<0$, when, as we have seen, the structure parameter adjusts slowly to the local conditions. In this case, $\Lambda_{1}$ may be obtained from (44) as

$$
\Lambda_{1}^{b}=\frac{\int_{0}^{2 \pi / \omega}\left|\frac{\partial u_{1}}{\partial \hat{y}}\right|^{c} \mathrm{~d} t}{\int_{0}^{2 \pi / \omega}\left|\frac{\partial u_{1}}{\partial \hat{y}}\right|^{a} \mathrm{~d} t} .
$$

In order to solve equations (39) and (48), we introduce an Ansatz motivated by the algebraic decay of velocity seen in the power-law case, namely

$$
u_{1}=c_{0} Y^{\alpha} \cos \left(\phi_{0} \log \left(Y / \hat{y}_{0}\right)-\omega t\right),
$$


where $Y=\hat{y}_{0}-\hat{y}$ for some $\hat{y}_{0}$ and where $c_{0}=\hat{y}_{0}^{-\alpha}$ to satisfy the boundary condition at $\hat{y}=0$. This solution is valid for $Y>0$, i.e. in the finite interval $0<\hat{y}<\hat{y}_{0}$.

Substituting the Ansatz (49) into equation (48) yields an expression for $\Lambda_{1}$,

$$
\Lambda_{1}^{b}=\left(c_{0} Y^{\alpha-1}\left(\alpha^{2}+\phi_{0}^{2}\right)^{1 / 2}\right)^{c-a} D_{0}
$$

where

$$
D_{0}=\frac{\int_{0}^{2 \pi / \omega}|\cos (\omega t)|^{c} \mathrm{~d} t}{\int_{0}^{2 \pi / \omega}|\cos (\omega t)|^{a} \mathrm{~d} t}=\frac{\int_{0}^{2 \pi}|\cos (\tau)|^{c} \mathrm{~d} t}{\int_{0}^{2 \pi}|\cos (\tau)|^{a} \mathrm{~d} \tau}
$$

and so

$$
\Lambda_{1}=\left[D_{0}\left(c_{0}\left(\alpha^{2}+\phi_{0}^{2}\right)^{1 / 2}\right)^{c-a}\right]^{1 / b} Y^{2}
$$

To find the constants $\alpha, \phi_{0}$ and $c_{0}$, equation (49) is substituted into (39) and

$$
\alpha=\frac{2 b+c-a}{c-a}
$$

is found by balancing the powers of $Y$, while $\phi_{0}$ and $c_{0}$ are found by balancing the coefficients of the $\sin$ and $\cos$ terms. The decay rate $\alpha$ is the same as for the fast-adjusting antithixotropic fluid.

The shear stress at the wall is given, to leading order, by

$$
\tau_{\mathrm{w}} \sim-\epsilon^{1+\beta / 2} D_{0}^{1 / b}\left(c_{0} \hat{y}_{0}^{\alpha-1}\right)^{(c-a+b) / b}\left(\alpha^{2}+\phi_{0}^{2}\right)^{(c-a) / 2 b}\left[\alpha \cos (\omega t)+\phi_{0} \sin (\omega t)\right] .
$$

It is straightforward to demonstrate that if the asymptotic expansion is carried to the next order then the structure parameter must be given by $\lambda \sim \epsilon^{\beta} \Lambda_{1}(y)+\epsilon^{2 b c /(2 b+c-a)} \Lambda_{2}(y, t)$, where

$$
\frac{\partial \Lambda_{2}}{\partial t}=-\left|\frac{\partial u_{1}}{\partial \hat{y}}\right|^{a} \Lambda_{1}^{b}+\left|\frac{\partial u_{1}}{\partial \hat{y}}\right|^{c} .
$$


This result will be of use when we check the validity of these solutions in section 4.1.4.

\subsubsection{Marginal behaviour when $\nu=0$}

The third and final case we consider is the marginal case $\nu=0$, in which all three terms in (10) are of comparable magnitude. Now, at leading order (40) becomes

$$
\frac{\partial \Lambda_{1}}{\partial t}=-\left|\frac{\partial u_{1}}{\partial \hat{y}}\right|^{a} \Lambda_{1}^{b}+\left|\frac{\partial u_{1}}{\partial \hat{y}}\right|^{c} .
$$

Physically, we may expect the behaviour to be intermediate between fastand slow- adjusting behaviour. In order to solve equations (39) and (56), two Ansätze are introduced, motivated by the behaviour of the fast- and slow- adjusting cases,

$$
u_{1}=Y^{\alpha} f(\tau) \text { and } \Lambda_{1}=Y^{2} g(\tau) \text {, }
$$

where $Y=\hat{y}_{0}-\hat{y}$ with $0 \leq \hat{y} \leq \hat{y}_{0}, \tau=\phi_{0} \log (Y)+\omega t+\tau_{0}, \tau_{0}$ is a phase difference, and the functions $f(\tau)$ and $g(\tau)$ are to be determined. Substituting equations (57) into the governing equations and balancing the powers of $y$ yields $\alpha=1+2 / c$, which in this case is identical to (53), together with the coupled equations

$$
\omega \frac{\partial g}{\partial \tau}=-\left|\alpha f+\phi_{0} \frac{\partial f}{\partial \tau}\right|^{a} g^{b}+\left|\alpha f+\phi_{0} \frac{\partial f}{\partial \tau}\right|^{c}
$$

and

$\omega \frac{\partial f}{\partial \tau}=\left(2 g+\phi_{0} \frac{\partial g}{\partial \tau}\right)\left(\alpha f+\phi_{0} \frac{\partial f}{\partial \tau}\right)+g\left[\alpha(\alpha-1) f+\phi_{0}(2 \alpha-1) \frac{\partial f}{\partial \tau}+\phi_{0}^{2} \frac{\partial^{2} f}{\partial \tau^{2}}\right]$.

These equations must be solved numerically, subject to the periodicity constraint $f(\tau)=f(\tau+2 \pi)$ and $g(\tau)=g(\tau+2 \pi)$ for all $\tau$. 
If the velocity imposed at the wall is precisely of the form $f(\tau)$ and has amplitude $\epsilon$, so the oscillation has dimensionless amplitude 1 at $y=0$, then $\hat{y}_{0}$ must be given by

$$
\left(\hat{y}_{0}-0\right)^{\alpha} f_{\max }=1, \quad \text { i.e. } \quad \hat{y}_{0}=f_{\max }^{-1 / \alpha},
$$

where $f_{\max }=\max _{\tau} f(\tau)$. We carry out numerical integrations to find $f$ and $g$. These functions always converge to a periodic solution, and the parameter $\phi_{0}$ is determined by a shooting method in order to set the period to be $2 \pi$ as required.

The shear stress at the wall is given, to leading order, by

$$
\tau_{\mathrm{w}} \sim-\epsilon^{1+\beta / 2} \hat{y}_{0}^{2 \alpha-1} g(\tau)\left(\alpha f(\tau)+\phi_{0} f^{\prime}(\tau)\right)
$$

\subsubsection{A posteriori estimate of the validity of the asymptotic solutions}

As in the case of a thixotropic fluid, the asymptotic solutions described in the preceding subsections have been obtained in the limit of small-amplitude oscillations, $\epsilon \rightarrow 0$ and depend on the additional independent parameter $\omega$. It is therefore useful to obtain a posteriori estimates of the validity of these solutions when $\omega$ is not necessarily of order unity.

From [24] we know that in the fast-adjusting regime $\hat{y}=O\left(\omega^{-1 /(n+1)}\right)$, and so $\partial u / \partial \hat{y}=O\left(\omega^{1 /(n+1)}\right)$. From equation (45), $\Lambda_{1}=O\left(\omega^{(c-a) /(2 b+c-a)}\right)$ and $\partial \Lambda_{1} / \partial t=O\left(\omega^{2(b+c-a) /(2 b+c-a)}\right)$, and hence, the validity condition for the asymptotic solution in the fast-adjusting case, namely

$$
\left|\epsilon^{\nu} \frac{\partial \Lambda_{1}}{\partial t}\right| \ll\left|\frac{\partial u_{1}}{\partial \hat{y}}\right|^{c}
$$

corresponds to

$$
\epsilon^{\nu} \omega^{2(b+c-a) /(2 b+c-a)} \ll \omega^{b c /(2 b+c-a)}, \quad \text { i.e. } \quad \epsilon^{\nu} \omega^{1+\nu / 2} \ll 1 .
$$


Fast-adjusting behaviour can therefore be expected in the asymptotic limits of either small oscillations, $\epsilon \rightarrow 0$, or slow oscillations, $\omega \rightarrow 0$.

In the slow-adjusting regime, equation (52) yields $\Lambda_{1}=O\left(\omega^{(c-a) /(2 b+c-a)}\right)$. To estimate the magnitude of $\partial \lambda / \partial t$ we need to consider the next order in $\epsilon$. Integrating equation (55) with respect to $\omega t$ implies that $\omega \Lambda_{2}=$ $O\left(\omega^{b c /(2 b+c-a)}\right)$; thus the validity condition for the asymptotic solution in the slow-adjusting case, namely

$$
\epsilon^{\beta} \Lambda_{1} \gg \epsilon^{2 b c /(2 b+c-a)} \Lambda_{2}
$$

corresponds to

$$
\epsilon^{\nu} \omega^{(c-a) /(2 b+c-a)} \gg \omega^{(b c-2 b-c+a) /(2 b+c-a)}, \quad \text { i.e. } \quad \epsilon^{\nu} \omega^{1+\nu / 2} \gg 1,
$$

which is exactly the complement of the condition (63) for fast adjustment

Figure 9 illustrates the parameter regimes in which the present asymptotic analysis suggests fast-adjusting, slow-adjusting and hysteretic behaviour. To avoid ambiguity, we will henceforth refer to the parameter regime $a<c$, $\nu>0$ as regime $\mathrm{A} 1$ and to the parameter regime $a<c, \nu<0$ as regime A2; we will reserve the terms 'fast-adjusting' and 'slow-adjusting' for the regimes, depending also on $\epsilon$ and $\omega$, in which the solutions display these behaviours. When the system is neither fast- nor slow-adjusting, we may expect its behaviour in general to be hysteretic, as shown in figure 9 .

\subsection{Numerical results for finite amplitude wall oscillations}

We now compare the asymptotic solutions for velocity, structure parameter and shear stress at the wall with numerical solutions calculated using the finite-element package Comsol 3.5a. As before, a finite numerical domain 
$0 \leq y \leq y_{\max }$ was used, with a domain length of $y_{\max }=10$ unless otherwise stated. The initial conditions were $u(y, 0)=0$ and $\lambda(y, 0)=0$ unless otherwise stated and all other technical details were as in section 3.2.

\subsubsection{Regime A1: $\nu>0$}

To illustrate the behaviour in regime A1, in which the structure parameter is fast-adjusting for small $\epsilon$, we take $a=1, b=3 / 10, c=2$ and $d=1$, giving $\nu=1 / 2$. We also set $\omega=1$ so that the condition (63) is easily satisfied.

We first consider small-amplitude oscillations with $\epsilon=0.01$. Figures $10 \mathrm{a}$ and $10 \mathrm{~b}$ show snapshots of the velocity $u(y, t)$ and structure parameter $\lambda(y, t)$ at equally spaced times during a period. The first snapshot in each figure is taken at $t=100$, by which time the system has adjusted to an essentially periodic state. The full numerical solution (solid lines) and the asymptotic solution (dashed lines) agree well for all values of $y$. In contrast to the corresponding thixotropic case, the antithixotropic velocity snapshots are very angular with 'corners' corresponding to the minima of $\lambda$ where the structure parameter, and thus the viscosity, is nearly zero. Also, as predicated by the asymptotic solution, the antithixotropic fluid displays a finite thickness boundary layer beyond which the fluid remains at rest, unaffected by the oscillating wall. When the viscosity has reached zero there is no diffusivity and there is effectively a slip layer at the edge of this boundary, across which no signal propagates [24].

To investigate the behaviour for larger oscillations, the amplitude was increased to $\epsilon=1$. Figures $10 \mathrm{c}$ and $10 \mathrm{~d}$ show snapshots of the velocity $u(y, t)$ and structure parameter $\lambda(y, t)$. There are no longer pronounced 'corners' in the velocity snapshots (figure $10 \mathrm{c}$ ), as the structure parameter no longer 
reduces to near zero at its minima (figure $10 \mathrm{~d}$ ). The velocity also decays more rapidly with distance from the wall than is predicted by the asymptotic solution. The magnitude of the structure parameter is significantly overestimated by the asymptotic solution because shear rates, and thus structure build up rates, are generally lower than predicted by the asymptotic solution.

When the amplitude is increased further to $\epsilon=10$ (figures 10 e and $\mathrm{f}$ ), these trends are still more strongly pronounced, and the asymptotic solution for small $\epsilon$ is clearly no longer valid. The structure now has almost completely built up near the wall (figure $10 \mathrm{f}$ ); however, it does not build up to the levels predicted by the asymptotic solution, and so the oscillations in the velocity die out more quickly than the asymptotic solution predicts (figure 10 e). Rather than having sharp spatial minima, the snapshots of $\lambda$ are now characterised by small oscillations around each minimum: these oscillations are the signature of hysteresis as the structure is unable to break down to zero as required by the instantaneous solution $\lambda_{\mathrm{av}}$. The effect of this on the shear stress at the wall will be discussed below.

\subsubsection{Regime A2: $\nu<0$}

To illustrate the behaviour in regime A2, in which the structure parameter is slow-adjusting for small $\epsilon$, we take $a=1, b=3 / 4, c=2$ and $d=1$, giving $\nu=-2 / 5$. We again set $\omega=1$ so that the condition (65) is easily satisfied.

We first consider small-amplitude oscillations with $\epsilon=0.01$. Figures $11 \mathrm{a}$ and $11 \mathrm{~b}$ show snapshots of the velocity $u(y, t)$ and structure parameter $\lambda(y, t)$ at equally spaced times during a period. The first snapshot is taken at $t=100$, by which time the velocity has adjusted to an essentially periodic state. The numerical solution (solid lines) and the asymptotic solution 
(dashed lines) agree well for all values of $y$. The velocity solution is not angular as in the fast-adjusting case. However, there is still a finite thickness boundary layer, at $0 \leq y \leq y_{0}=\epsilon^{2 / 5} \hat{y}_{0} \approx 0.586$. In figure $11 \mathrm{~b}$ the numerical solution (solid lines) for the structure parameter oscillates weakly over a period, around the asymptotic value (52).

To investigate the behaviour for larger oscillations, the amplitude was again increased to $\epsilon=1$. Figures $11 \mathrm{c}$ and $\mathrm{d}$ show snapshots of the velocity $u(y, t)$ and structure parameter $\lambda(y, t)$. The deviation from the asymptotic solution is now more apparent. The structure parameter now oscillates around an average equilibrium value, and the asymptotic solution overestimates this. Figure $11 \mathrm{c}$ shows the velocity decaying faster than the asymptotic solution predicts, as the viscosity is lower than predicted.

When the amplitude is increased further to $\epsilon=10$ (figure 11 e and $\mathrm{f}$ ), the asymptotic solution for small $\epsilon$ is clearly no longer valid. The behaviour for $\epsilon=10$ is very different from that in the limit $\epsilon \rightarrow 0$. However, the structure parameter still varies only weakly throughout a period (figure $11 \mathrm{f}$ ) so the system still shows essentially slow-adjusting behaviour. The velocity now decays much faster than the asymptotic solution predicts. As $\epsilon$ increases, figures $11 \mathrm{~b}, \mathrm{~d}$ and e tell a similar story to that seen in figure 10. As $\epsilon$ increases, first oscillations and then small double oscillations appear: the numerical solution in figure $11 \mathrm{f}$ is very similar to that in figure $10 \mathrm{f}$. As in the thixotropic case, with increasing $\epsilon$ the distinction between the regimes becomes increasingly blurred. 


\subsubsection{Shear stress at the wall}

It is also of interest to examine the shear stress at the wall. Figures 12 a and $\mathrm{b}$ show the shear stress $\tau_{\mathrm{w}}(t)$, normalised by $\epsilon^{1+\beta / 2}$, for regimes $\mathrm{A} 1$ and A2 respectively. (In figure 12 a for $\epsilon=10$ only, the mesh comprised 1920 equally-spaced grid points to resolve the small-scale oscillations properly.)

In each case, the variation of $\tau_{\mathrm{w}}(t)$ for small $\epsilon$ is nearly sinusoidal, and, in contrast to the thixotropic case, as $\epsilon$ is increased, the variation remains nearly sinusoidal. In both figures, the rescaled shear stress $\tau_{\mathrm{w}} / \epsilon^{1+\beta / 2}$ decreases with increasing $\epsilon$, although it decreases more gradually in regime A2 as shown in figure $12 \mathrm{~b}$.

It is interesting to note that the rapid variations of $\tau_{\mathrm{w}}$ seen for thixotropic fluids (figure 6) are absent for antithixotropic cases, because there is no longer a positive feedback between $\lambda$ and $\partial u / \partial y$ at the wall.

\subsubsection{Marginal regime $\nu=0$}

Finally, to illustrate the behaviour in the marginal regime $\nu=0$ we take $a=1, b=1 / 2, c=2$ and $d=1$, giving $\nu=0, \alpha=2$ and the numerically determined asymptotic parameters $\hat{y}_{0}=3.15$ and $\phi_{0}=2.591$. As in all the previously considered regimes, we set $\omega=1$.

We first consider small-amplitude oscillations with $\epsilon=0.01$. Figures $13 \mathrm{a}$ and $13 \mathrm{~b}$ show snapshots of the velocity $u(y, t)$ and structure parameter $\lambda(y, t)$ at equally spaced times during a period. The first snapshot in each figure is taken at $t=100$, by which time the system has adjusted to an essentially periodic state. Although the asymptotic solutions capture the overall decay of the velocity and structure parameter, they deviate from the numerical solutions close to the wall $y=0$; this reflects the inconsistency 
between the non-sinusoidal velocity variation in the asymptotic solution and the sinusoidal variation imposed on the numerical solution (cf. [24]).

To investigate the behaviour for larger oscillations, the amplitude was again increased first to $\epsilon=1$ (figures $13 \mathrm{c}$ and $\mathrm{d}$ ) and then to $\epsilon=10$ (figure 13 e and $\mathrm{f}$ ). As $\epsilon$ is increased, the asymptotic solutions come to severely overestimate the numerical solutions for both the velocity and structure parameter, and by $\epsilon=10$, double oscillations of the structure parameter have again appeared (figure $13 \mathrm{f}$ ). Figures $10 \mathrm{f}, 11 \mathrm{f}$ and $13 \mathrm{f}$ all show the same hysteretic behaviour: distinctive fast and slow behaviour is no longer apparent.

The shear stress in the marginal regime (figure 14) is also similar to that in the other two regimes and the variation is nearly sinusoidal for all values of $\epsilon$ examined. The rescaled shear stress $\tau_{\mathrm{w}} / \epsilon^{1+\beta / 2}$ decreases weakly with increasing $\epsilon$. The agreement between numerical and asymptotic solutions for $\epsilon=0.01$ is poorer than before, reflecting the poorer agreement for $u$ and $\lambda$ (figures $13 \mathrm{a}$ and $\mathrm{b}$ ).

\section{Rheograms for thixotropic and antithixotropic fluids}

A standard form in which to present rheometric data is a plot of the shear stress at the wall against the shear rate in the rheometer, often called a rheogram. Although the Stokes flows analysed in this paper are nonrheometric, because the shear rate is not spatially constant, it is interesting to construct equivalent rheograms in order to identify key features of the flow. In particular, hysteresis loops in a rheogram are the signature of lag effects [2]. 
In this section, we present the results of the numerical integrations presented in sections 3 and 4 in this form. In each case, the shear stress $\tau_{\mathrm{w}}$ is plotted against the instantaneous shear rate $\partial u / \partial y$ evaluated at the wall, $y=0$. In each plot, an initial transient can be seen followed by adjustment to an effectively periodic state: we focus our discussion on these periodic states. The rheograms also plot the instantaneous equilibrium shear stress $\tau_{\text {eq }}=\lambda_{\text {eq }} \partial u / \partial y$, where $\lambda_{\text {eq }}$ is calculated from equation (12).

\subsection{Rheograms for thixotropic fluids}

Figure 15 shows rheograms for the three thixotropic regimes: regime T1 (figures 15 a and b, which correspond to the shapshots in figure 3); the marginal regime (figures $15 \mathrm{c}$ and $\mathrm{d}$, which correspond to the shapshots in figure 7); and regime T2 (figures 15 e and f, which correspond to the shapshots in figure 4$)$. The corresponding plots of $\tau_{\mathrm{w}}(t)$ are shown in figures 6 and 8 . In each case the equilibrium behaviour is shear-thinning, and $c-a+b=$ 0 so $\tau_{\text {eq }} \rightarrow 1$ as $|\partial u / \partial y| \rightarrow \infty$ (section 2.4 ).

For small values of $\epsilon$ (figures 15 a, c and e), the fluid is nearly fully structured, with $\lambda \approx 1$. The rheograms are therefore indistinguishable from those for a Newtonian fluid, $\tau_{\mathrm{w}}= \pm|\partial u / \partial y|$. Note also that since $\lambda_{\mathrm{eq}} \approx$ $\lambda_{\mathrm{av}} \approx 1$, there is no visible difference between the fast- and slow-adjusting regimes.

As $\epsilon$ increases, hysteresis loops appear, as shown for $\epsilon=10$ (figures $15 \mathrm{~b}$, $\mathrm{d}$ and $\mathrm{f}$ ). The rapid variation of $\tau_{\mathrm{w}}$ at small shear rates, commented on in section 3.2.2, manifests itself as a small secondary loop close to the origin in these plots; note that the curve does not intersect itself so this secondary loop is not completely closed. The overall behaviour is reminiscent of the 
stress overshoot due to the breakdown of an initial structure represented in figure 2(b) of Mewis \& Wagner [2]. Indeed, $\tau_{\mathrm{w}}$ is everywhere higher than or equal to its 'equilibrium' value $\tau_{\text {eq }}$, so we may think of these hysteresis loops as representing the signature of a persistent stress overshoot.

For the large value of $\epsilon$ employed in figures $15 \mathrm{~b}, \mathrm{~d}$ and $\mathrm{f}$, some systematic differences are apparent between the three regimes: as $d$ increases from regime $\mathrm{T} 1$ through to regime $\mathrm{T} 2$, the amplitude of variation of $\tau_{\mathrm{w}}$ decreases, so the hysteresis loops become narrower. It is slightly surprising that the deviation of $\tau_{\mathrm{w}}$ from $\tau_{\text {eq }}$ is greatest in the 'fast' regime $\mathrm{T} 1$ and least in the 'slow' regime T2.

\subsection{Rheograms for antithixotropic fluids}

Figure 16 shows rheograms for the three antithixotropic regimes: regime A1 (figures 16 a and b, which correspond to the shapshots in figure 10); the marginal regime (figures $16 \mathrm{c}$ and $\mathrm{d}$, which correspond to the shapshots in figure 13); and regime A2 (figures 16 e and f, which correspond to the shapshots in figure 11). The corresponding plots of $\tau_{\mathrm{w}}(t)$ are shown in figures 12 and 14.

In contrast to the thixotropic case, the three regimes behave differently even for $\epsilon=0.01$. Hysteresis loops are present in all three regimes; in regime A2 (figure 16 e) we see an additional consequence of slow adjustment, which is that the amount of hysteresis decays over many cycles, with the loops gradually moving outwards and becoming narrower as the system approaches its periodic state. In the fast-adjusting regime only (figure 16 a), the hysteresis loops are centred on the equilibrium curve $\tau_{\text {eq }}$; in the slow-adjusting

regime (figure 16 e) the equilibrium solution is clearly irrelevant, whereas the 
marginal regime (figure 16 c) may be affected by the competition between the sinusoidal forcing at the wall and the 'natural' non-sinusoidal waveform of oscillation (cf. figure 13 a). As $b$ is increased from regime A1 through to regime $\mathrm{A} 2$, it is also noticeable that the range of shear rates decreases somewhat while the range of shear stresses increases.

Again in contrast to the thixotropic case, the rheograms for large $\epsilon$ (figure $16 \mathrm{~b}, \mathrm{~d}$ and $\mathrm{f}$ ) are almost indistinguishable in the three antithixotropic regimes, which is consistent with the general similarity of the solutions (section 4.2.4). Although some hysteresis is apparent, these plots in fact closely resemble those that would be expected for a Newtonian fluid with a constant value of $\lambda=1$, reflecting the fact that the structure is nearly completely built up at the wall. As for a thixotropic fluid under small oscillations, $\lambda_{\mathrm{eq}} \approx \lambda_{\mathrm{av}} \approx 1$ and so the equilibrium shear stress $\tau_{\text {eq }}$ agrees rather closely with the numerically calculated stress; there are, however, still some underand overshoots at lower shear rates.

In summary, although the rheograms reveal interesting patterns in the behaviour of the solutions, it is not straightforward to extract distinctive signatures of thixotropic or antithixotropic behaviour from them. This sounds a cautionary note for future attempts to use Stokes flow as a non-rheometric rheological device [22].

\section{Summary and conclusions}

We have described a combined asymptotic and numerical investigation of the Stokes problem for a general model of thixotropic and antithixotropic fluids. The emphasis of our study has been on the periodic oscillatory be- 
haviour of the system, rather than on the transient initial phase in which the system adjusts to the attracting periodic solution.

The asymptotic analysis in the limit of small-amplitude oscillations, $\epsilon \rightarrow$ 0 , revealed distinct regimes of fast and slow structural response for both thixotropic and antithixotropic fluids. In the fast-adjusting regimes, the structure is an instantaneous function of the shear, and the fluid acts like a generalised Newtonian fluid. In the slow-adjusting regimes, the local structure is determined by a long-term average of build-up and breakdown rates. In the marginal regimes between the limits of fast and slow behaviour, the variation of the structure parameter is lagged and attenuated relative to its instantaneous equilibrium value. This lagged and attenuated response becomes increasingly dominant in all regimes as $\epsilon$ increases. When $\epsilon$ is large enough the distinction between the fast- and slow-adjusting regimes disappears entirely.

The boundaries of the fast- and slow-adjusting regimes depend on the relative magnitudes of the dimensionless amplitude of oscillation $\epsilon$ and the dimensionless frequency of oscillation $\omega$. They also depend, in a rather subtle manner, on the exponents $a, b, c$ and $d$ that appear in the evolution equation for the structure parameter. This subtlety suggests that it may be necessary when employing structure evolution equations to pay careful attention to these exponents. Such attention would contrast with the prevailing approach, which is to choose these exponents for simplicity and to devote most attention to identifying a suitable constitutive equation.

The most interesting deviations from Newtonian behaviour come for antithixotropic fluids, which, like shear-thickening power-law fluids [24], develop 
a boundary layer of finite thickness beyond which the fluid is unaffected by the oscillating wall. The thickness of this layer scales with $\epsilon^{\beta / 2}$, where the parameter $\beta$ is defined by (41) in terms of the exponents in the structure evolution equation; the velocity amplitude decays algebraically and the structure parameter decays quadratically towards the edge of the layer. In contrast to the internal behaviour of the fluid, the shear stress at the wall, $\tau_{\mathrm{w}}(t)$, shows surprisingly weak deviations from Newtonian behaviour. In particular, changes in the structure and the shear rate close to the wall compensate so that for thixotropic fluids the shear stress scales roughly with $\epsilon$ even when $\epsilon$ is significantly larger than unity; for antithixotropic fluids, similarly, the shear stress scales roughly with $\epsilon^{1+\beta / 2}$ well beyond the regime in which this scaling is formally valid.

Although Stokes flow proves to be a revealing test of rheological models, it is not straightforward to identify the rheological characteristics of the fluid from stress-shear plots (rheograms). However, such plots do reveal hysteresis clearly in many cases, and also emphasise some more complex behaviour such as the feedback between increasing structure and decreasing shear that occurs during some phases of the oscillation for thixotropic fluids.

This investigation of Stokes flow - one of the simplest classical boundaryvalue problems for a viscous fluid - has revealed the surprisingly subtle and complex nature of the response of structured fluids. We suggest that similar studies of other apparently simple problems could usefully expand the scientific understanding of these fluids.

Acknowledgements. C. R. McA. is supported by a Doctoral Training 
Award funded by the Engineering and Physical Sciences Research Council. Part of this work was undertaken while S. K. W. was a Visiting Fellow in the Department of Mechanical and Aerospace Engineering in the School of Engineering and Applied Science at Princeton University, USA, and part was undertaken while he was a Visiting Fellow in the Oxford Centre for Collaborative Applied Mathematics (OCCAM) at the University of Oxford, England. This publication was based on work supported in part by Award No KUKC1-013-04, made by King Abdullah University of Science and Technology (KAUST). We are also grateful to Dr Michele Taroni (OCCAM, Oxford) and Prof. Iain W. Stewart (Strathclyde) for their valuable suggestions on aspects of this work.

\section{Appendix A. Pseudo-Newtonian behaviour when $a=c$}

In this Appendix we consider the special case $a=c$ in which the equilibrium value of the structure parameter $\lambda$ is independent of the shear rate: for reasons which will shortly become apparent this will be termed 'pseudoNewtonian' behaviour.

In this special case, the characteristic scales (8) are undefined and we instead non-dimensionalise using the frequency of oscillation $\hat{\omega}$, obtaining

$$
\hat{U}_{0}=\hat{\nu}_{0}^{1 / 2}\left(\frac{\hat{\omega}^{2-a}}{\hat{k}_{1}^{2}}\right)^{1 /(2 a)}, \quad \hat{Y}_{0}=\left(\frac{\hat{\nu}_{0}}{\hat{\omega}}\right)^{1 / 2} \quad \text { and } \quad \hat{T}_{0}=\frac{1}{\hat{\omega}} .
$$

Equation (9) is unchanged, while equation (7) becomes

$$
\frac{\partial \lambda}{\partial t}=-\left|\frac{\partial u}{\partial y}\right|^{a}\left[\lambda^{b}-\kappa_{2}(1-\lambda)^{d}\right]
$$


where $\kappa_{2}=\hat{k}_{2} / \hat{k}_{1}$ is the ratio of the structural build-up and breakdown rates. Since the frequency of oscillation has been scaled out, the wall boundary condition becomes $u(0, t)=\epsilon \cos (t)$.

Equations (9) and (A.2) admit a periodic exact solution for all values of $\epsilon$

$$
u=\epsilon \mathrm{e}^{-\gamma y} \cos (\gamma y-t) \quad \text { where } \quad \gamma=\sqrt{\frac{1}{2 \lambda}}
$$

and where the constant value of $\lambda$ satisfies

$$
\frac{\lambda^{b}}{(1-\lambda)^{d}}=\kappa_{2}
$$

The periodic behaviour of the system is therefore identical to that of a Newtonian fluid, but with a viscosity that is self-selected via the equilibrium value of the structure parameter rather than being independently imposed.

Although the local shear rate does not control the eventual value of $\lambda$, the shear rate determines how rapidly the pseudo-Newtonian solution is reached from a given initial condition. Figure 17 illustrates the adjustment of $\lambda(y, t)$ from the initial condition $\lambda(y, 0)=1$. Since $\partial \lambda / \partial t \propto|\partial u / \partial y|^{a}$, adjustment is most rapid close to the wall, where the pseudo-Newtonian solution becomes established within a few periods; beyond a distance of order $1 / \gamma$, however, adjustment is far slower, and at the right-hand end of the computational domain the pseudo-Newtonian solution is attained only at extremely large times.

\section{References}

[1] H. A. Barnes, Thixotropy - a review, Journal of Non-Newtonian Fluid Mechanics 70 (1997) 1-33. 
[2] J. Mewis, N. J. Wagner, Thixotropy, Advances in Colloid and Interface Science 147-148 (2009) 214-227.

[3] F. Bautista, J. M. de Santos, J. E. Puig, O. Manero, Understanding thixotropic and antithixotropic behaviour of viscoelastic micellar solutions and liquid crystalline dispersions. I. The model, Journal of NonNewtonian Fluid Mechanics 80 (1999) 93-113.

[4] E. S. Boek, J. T. Padding, V. J. Anderson, P. M. J. Tardy, J. P. Crawshaw, J. R. A. Pearson, Constitutive equations for extensional flow of wormlike micelles: stability analysis of the Bautista-Manero model, Journal of Non-Newtonian Fluid Mechanics 126 (2005) 39-46.

[5] J. D. Sherwood, Transient flow of viscoelastic, thixotropic fluid in a vane rheometer or infinite slot, Journal of Non-Newtonian Fluid Mechanics 154 (2008) 109-119.

[6] D. Pritchard, J. R. A. Pearson, Viscous fingering of a thixotropic fluid in a porous medium or a narrow fracture, Journal of Non-Newtonian Fluid Mechanics 135 (2006) 117-127.

[7] P. R. de Souza Mendes, Modeling the thixotropic behavior of structured fluids, Journal of Non-Newtonian Fluid Mechanics 164 (2009) 66-75.

[8] K. L. Maki, Y. Renardy, The dynamics of a simple model for a thixotropic yield stress fluid, Journal of Non-Newtonian Fluid Mechanics 165 (2010) 1373-1385.

[9] P. Coussot, Q. D. Nguyen, H. T. Huynh, D. Bonn, Avalanche behaviour in yield stress fluids, Physical Review Letters 88 (2002) 175501. 
[10] N. Roussel, R. Le Roy, P. Coussot, Thixotropy modelling at local and macroscopic scales, Journal of Non-Newtonian Fluid Mechanics 117 (2004) 85-95.

[11] H. T. Huynh, N. Roussel, P. Coussot, Aging and free surface flow of a thixotropic fluid, Physics of Fluids 17 (2005) 033101.

[12] H. Chanson, S. Jarny, P. Coussot, Dam break wave of thixotropic fluid, Journal of Hydraulic Engineering 132 (2006) 280-293.

[13] W. Liu, K.-Q. Zhu, A study of start-up flow of thixotropic fluids including inertia effects on an inclined plane, Physics of Fluids 23 (2011) 013103.

[14] J. Billingham, J. W. J. Ferguson, Laminar, unidirectional flow of a thixotropic fluid in a circular pipe, Journal of Non-Newtonian Fluid Mechanics 47 (1993) 21-55.

[15] J. J. Derksen, Prashant, Simulations of complex flow of thixotropic liquids, Journal of Non-Newtonian Fluid Mechanics 160 (2009) 65-75.

[16] F. Moore, The rheology of ceramic slips and bodies, Transactions and Journal of the British Ceramic Society 58 (1959) 470-494.

[17] H. A. Ardakani, E. Mitsoulis, S. G. Hatzikiriakos, Thixotropic flow of toothpaste through extrusion dies, Journal of Non-Newtonian Fluid Mechanics 166 (2011) 1262-1271.

[18] K. Najmi, M. Karinzadeh, K. Sedeghy, Lubricating flow of thixotropic fluids in slipper-pad bearing: a numerical study, Nihon Reoroji 
Gakkaishi (Journal of the Society of Rheology, Japan) 39 (2011) 153158.

[19] S. Livescu, R. V. Roy, L. W. Schwartz, Leveling of thixotropic liquids, Journal of Non-Newtonian Fluid Mechanics 166 (2011) 395-403.

[20] A. Potanin, 3D simulations of the flow of thixotropic fluids, in largegap Couette and vane-cup rheometers, Journal of Non-Newtonian Fluid Mechanics 165 (2010) 299-312.

[21] P. G. Drazin, N. Riley, The Navier-Stokes equations: a classification of flows and exact solutions, number 334 in London Mathematical Society Lecture Notes Series, Cambridge University Press, 2006.

[22] N. J. Balmforth, Y. Forterre, O. Pouliquen, The viscoplastic Stokes layer, Journal of Non-Newtonian Fluid Mechanics 158 (2009) 46-53.

[23] L. Ai, K. Vafai, An investigation of Stokes' second problem for nonNewtonian fluids, Numerical Heat Transfer Part A - Applications 47 (2005) 955-980.

[24] D. Pritchard, C. R. McArdle, S. K. Wilson, The Stokes boundary layer for a power-law fluid, Journal of Non-Newtonian Fluid Mechanics 166 (2011) 745-753.

[25] K. R. Rajagopal, A note on unsteady unidirectional flows of a nonNewtonian fluid, International Journal of Non-Linear Mechanics 17 (1982) 369-373. 
[26] K. R. Rajagopal, T. Y. Na, On Stokes' problem for a non-Newtonian fluid, Acta Mechanica 48 (1983) 233-239.

[27] T. Hayat, S. Asghar, A. M. Siddiqui, Stokes' second problem for a Johnson-Segalman fluid, Applied Mathematics and Computation 148 (2004) 697-706.

[28] M. Khan, A. Anjum, C. Fetecau, On exact solutions of Stokes' second problem for a Burgers fluid, I. The case $\gamma<\lambda^{2} / 4$, Zeitschrift für angewandte Mathematik und Physik 61 (2010) 697-720. 


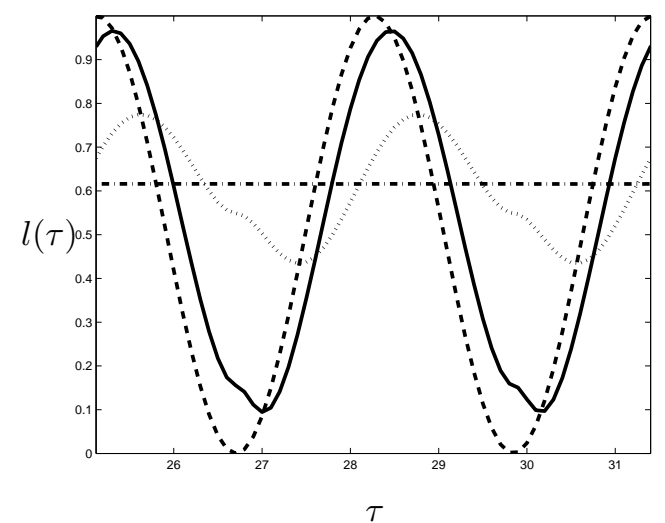

Figure 1: Periodic solutions to (30) for the structure waveform $l(\tau)$ with $a=2, b=1, c=1$, $d=1 / 2$ and $\omega=0$ (dashed; equation (32)); $\omega=0.1$ (solid); $\omega=0.5$ (dotted); $\omega=1000$ (dotdashed). 


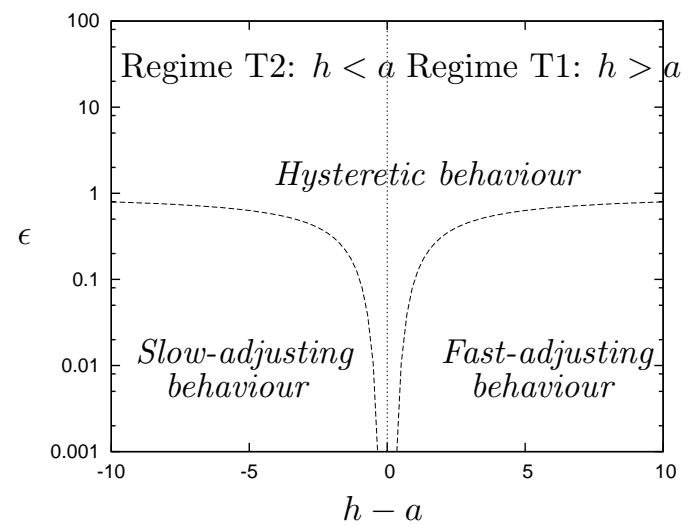

Figure 2: Outline of regimes of validity for the asymptotic solutions for a thixotropic fluid. The dashed lines represent indicative regime boundaries for $\omega=1: \epsilon^{h-a}=10$ for $h-a<0$ and $\epsilon^{h-a}=0.1$ for $h-a>0$. The dotted line represents the marginal regime $h=a$. 

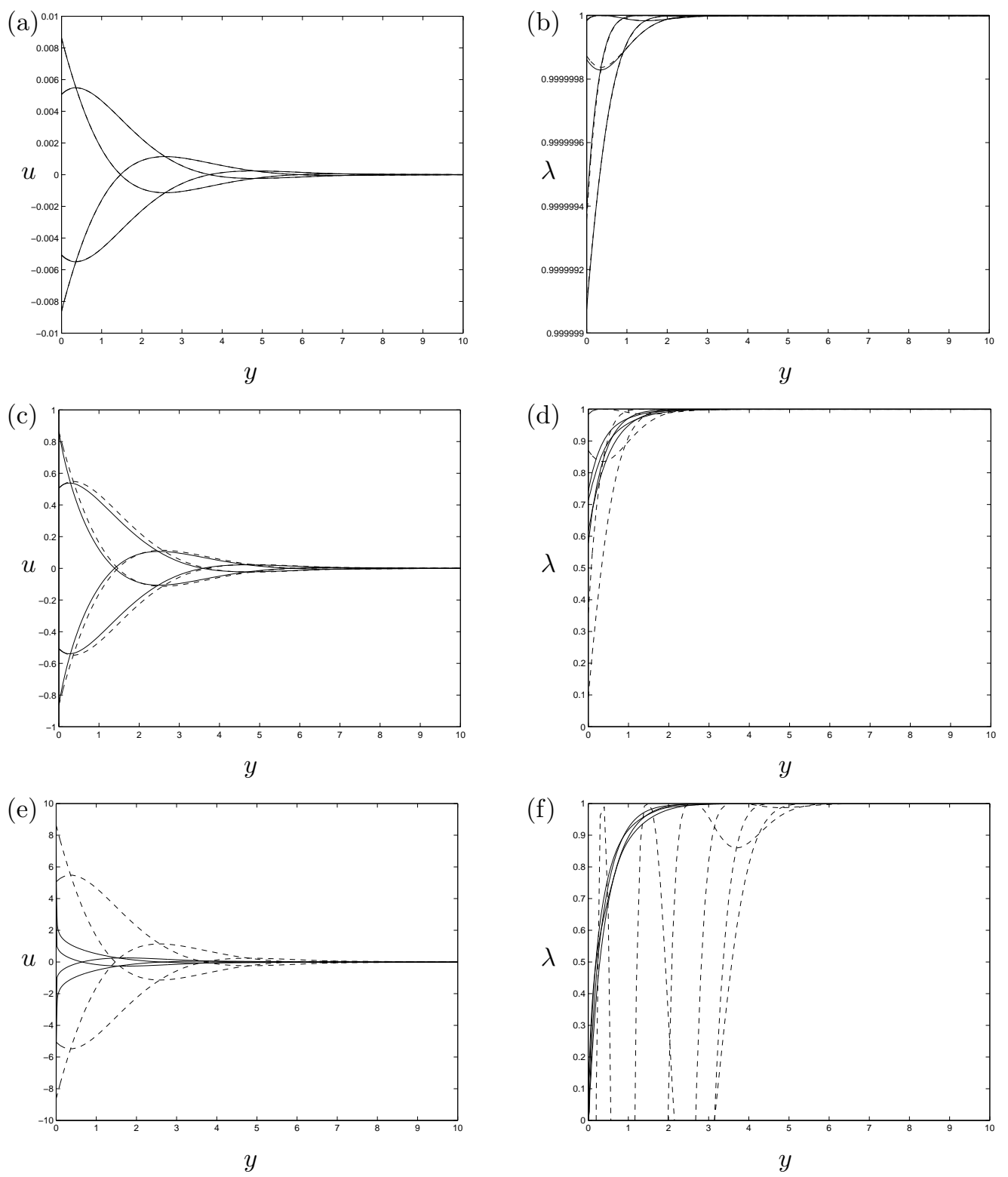

Figure 3: Thixotropic regime T1 $(h>a)$ : snapshots of the numerical solutions (solid lines) and fast-adjusting asymptotic solutions (dashed lines) with $a=2, b=1, c=1, d=1 / 3$, $\omega=1$ and $(\mathrm{a}, \mathrm{b}) \epsilon=0.01 ;(\mathrm{c}, \mathrm{d}) \epsilon=1 ;(\mathrm{e}, \mathrm{f}) \epsilon=10$. Plots (a), (c) and (e) show the velocity $u(y, t)$ at $t=100+n \pi / 2$ for $n=0,1,2$ and 3 ; the asymptotic solution is given by (17). Plots (b), (d) and (f) show the structure parameter $\lambda(y, t)$ at $t=100+n \pi / 4$ for $n=0,1,2,3 ;$ the asymptotic solution is gigen by $(22)$. 

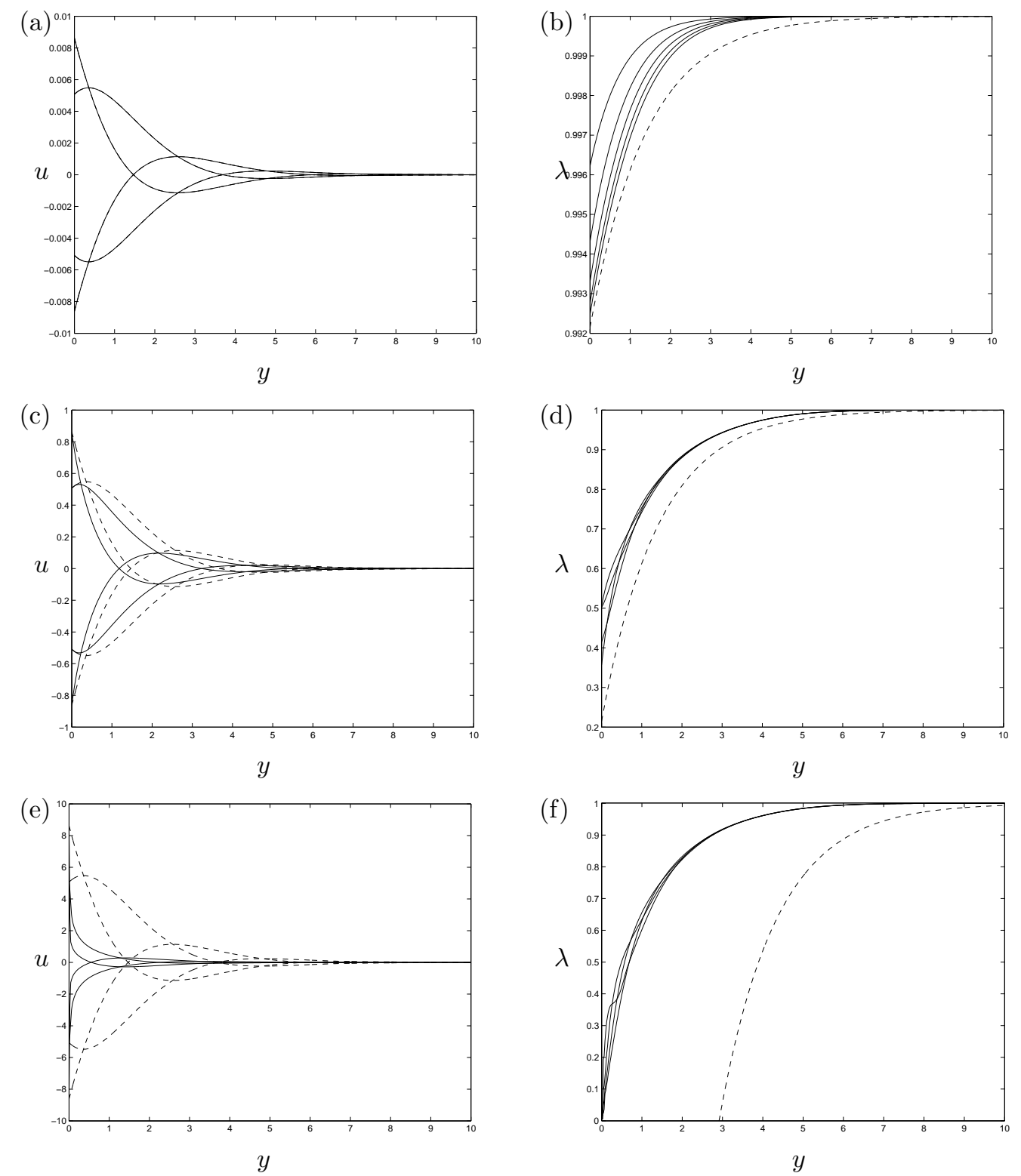

Figure 4: Thixotropic regime T2 $(h<a)$ : snapshots of the numerical solutions (solid lines) and slow-adjusting asymptotic solutions (dashed lines) with $a=2, b=1, c=1, d=1$, $\omega=1$ and $(\mathrm{a}, \mathrm{b}) \epsilon=0.01 ;(\mathrm{c}, \mathrm{d}) \epsilon=1 ;(\mathrm{e}, \mathrm{f}) \epsilon=10$. Plots (a), (c) and (e) show the velocity $u(y, t)$ at $t=100+n \pi / 2$ for $n=0,1,2$ and 3 ; the asymptotic solution is given by (17). Plot (b) shows the structure parameter $\lambda(y, t)$ at $t=100,200,300,400$ and 500, while plots $(\mathrm{d})$ and (f) show the structure parameter $\lambda(y, t)$ at $t=100+n \pi / 4$ for $n=0$, $1,2,3$; the asymptotic solution is given by (24). 

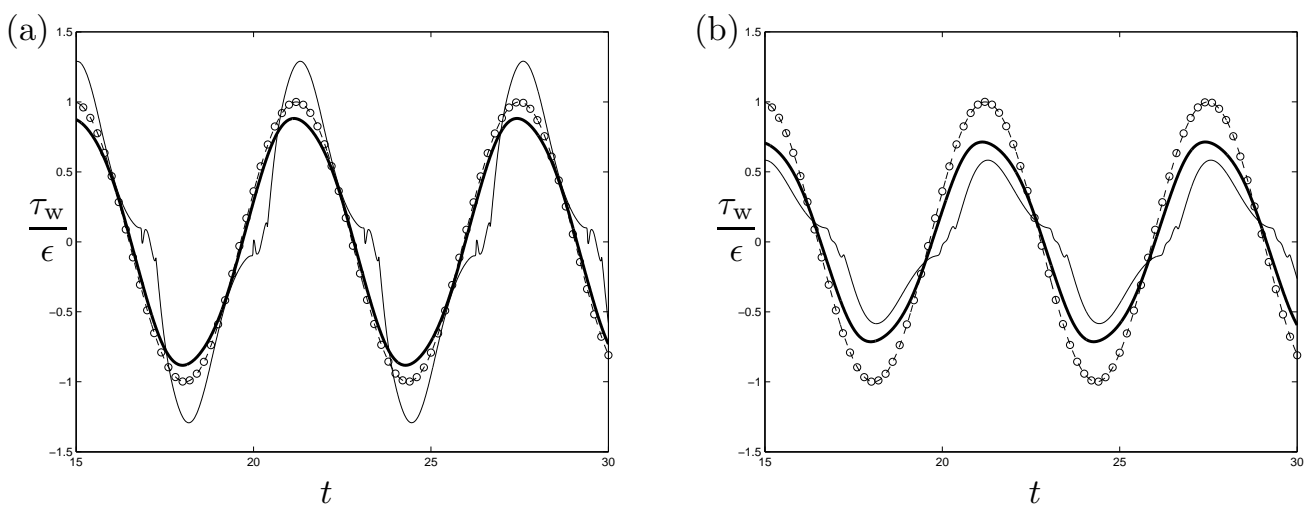

Figure 5: Normalised shear stress at the wall, $\tau_{\mathrm{w}}(t) / \epsilon$, with $a=2, b=1, c=1$ and $\omega=1$ and (a) $d=1 / 3$, (b) $d=1$. Figure (a) is the shear stress for regime T1 $(h>a)$ and figure (b) is the shear stress for regime T2 $(h<a)$. The circles represent the asymptotic solution; lines represent the numerical solutions for $\epsilon=0.01$ (dashed); $\epsilon=1$ (thick solid); $\epsilon=10$ (thin solid). 

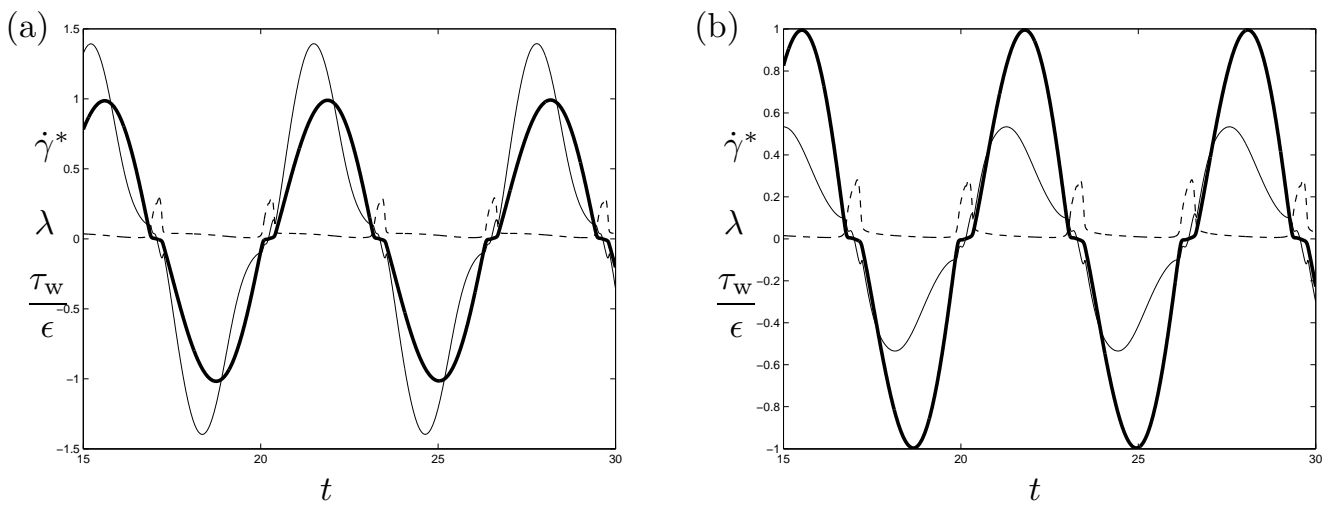

Figure 6: Normalised shear stress, normalised shear rate and structure parameter evaluated at the wall with $a=2, b=1, c=1$ and $\omega=1$ and (a) $d=1 / 3$, (b) $d=1$. Figure (a) shows regime $\mathrm{T} 1(h>a)$ and figure $(\mathrm{b})$ shows regime $\mathrm{T} 2(h<a)$. Lines represent the numerical solutions for $\tau_{\mathrm{w}} / \epsilon$ (thin solid); $\dot{\gamma}^{*}=(\partial u / \partial y) / \max (\partial u / \partial y)$ (thick solid); $\lambda$ (dashed). 

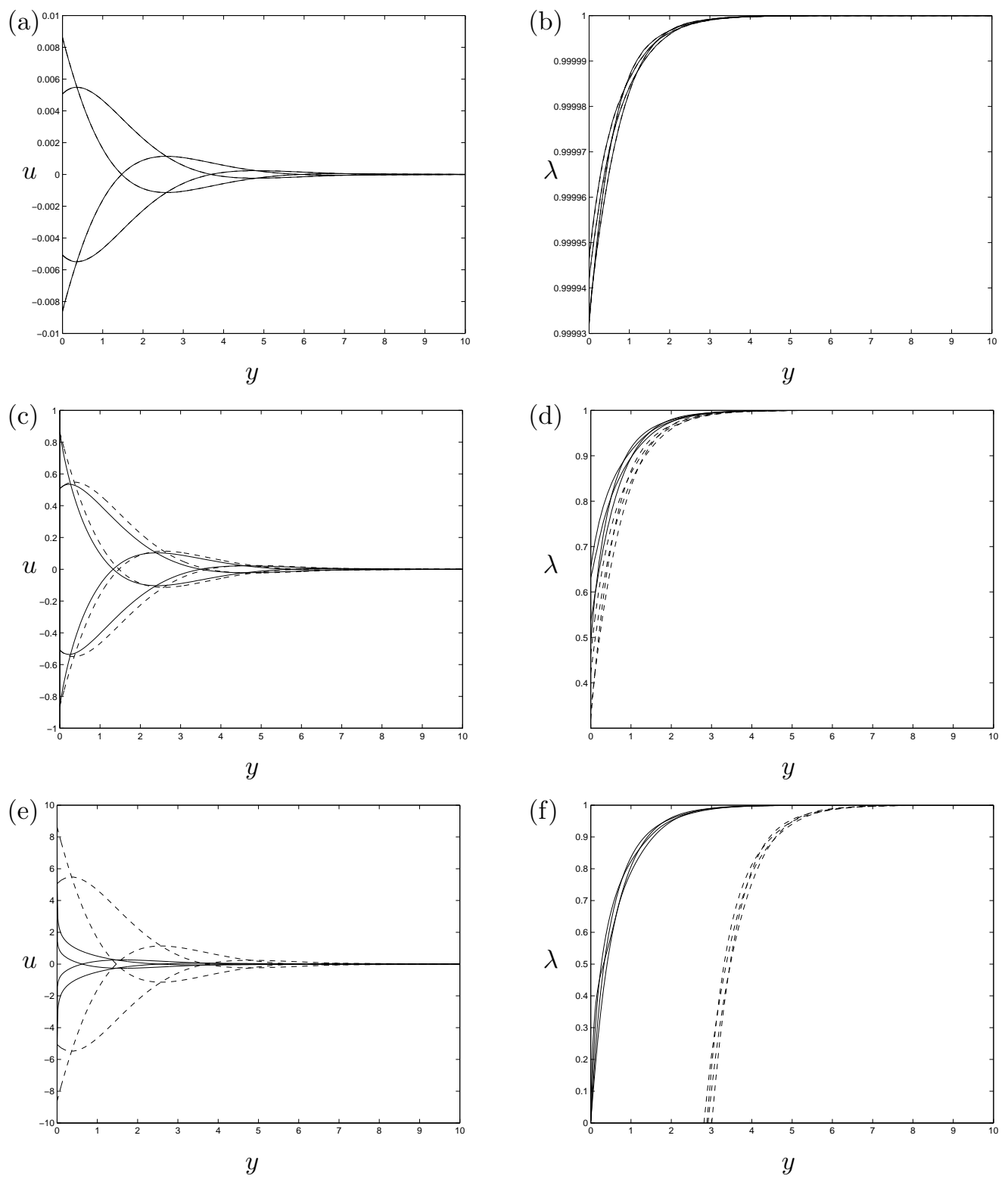

Figure 7: Thixotropic marginal regime $(h=a)$ : snapshots of the numerical solutions (solid lines) and marginal asymptotic solutions (dashed lines) with with $a=2, b=1, c=1$, $d=1 / 2, \omega=1$ and $(\mathrm{a}, \mathrm{b}) \epsilon=0.01 ;(\mathrm{c}, \mathrm{d}) \epsilon=1 ;(\mathrm{e}, \mathrm{f}) \epsilon=10$. Plots (a), (c) and (e) show the velocity $u(y, t)$ at $t=100+n \pi / 2$ for $n=0,1,2$ and 3 ; the asymptotic solution is given by (17). Plots (b), (d) and (f) show the structure parameter $\lambda(y, t)$ at $t=100+n \pi / 4$ for $n=0,1,2,3 ;$ the asymptotic solution is gigen by $(31)$. 


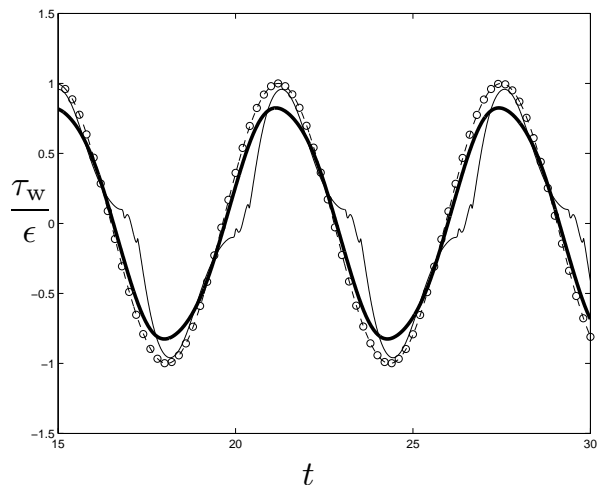

Figure 8: Thixotropic marginal regime: normalised shear stress at the wall, $\tau_{\mathrm{w}}(t) / \epsilon$, with $a=2, b=1, c=1, d=1 / 2$ and $\omega=1$. The circles represent the asymptotic solution (23); lines represent the numerical solutions for $\epsilon=0.01$ (dashed); $\epsilon=1$ (thick solid); $\epsilon=10$ (thin solid). 


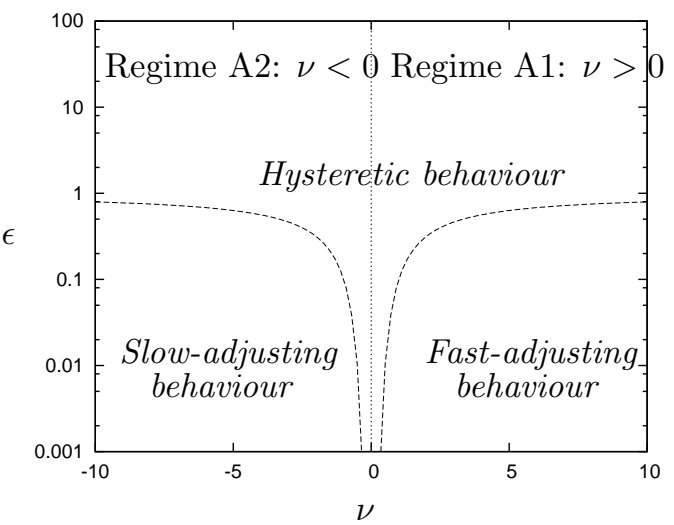

Figure 9: Outline of regimes of validity for the asymptotic solutions for an antithixotropic fluid. The dashed lines represent indicative regime boundaries for $\omega=1$ : $\epsilon^{\nu}=10$ for $\nu<0$ and $\epsilon^{\nu}=0.1$ for $\nu>0$. The dotted line represents the marginal regime $\nu=0$. 

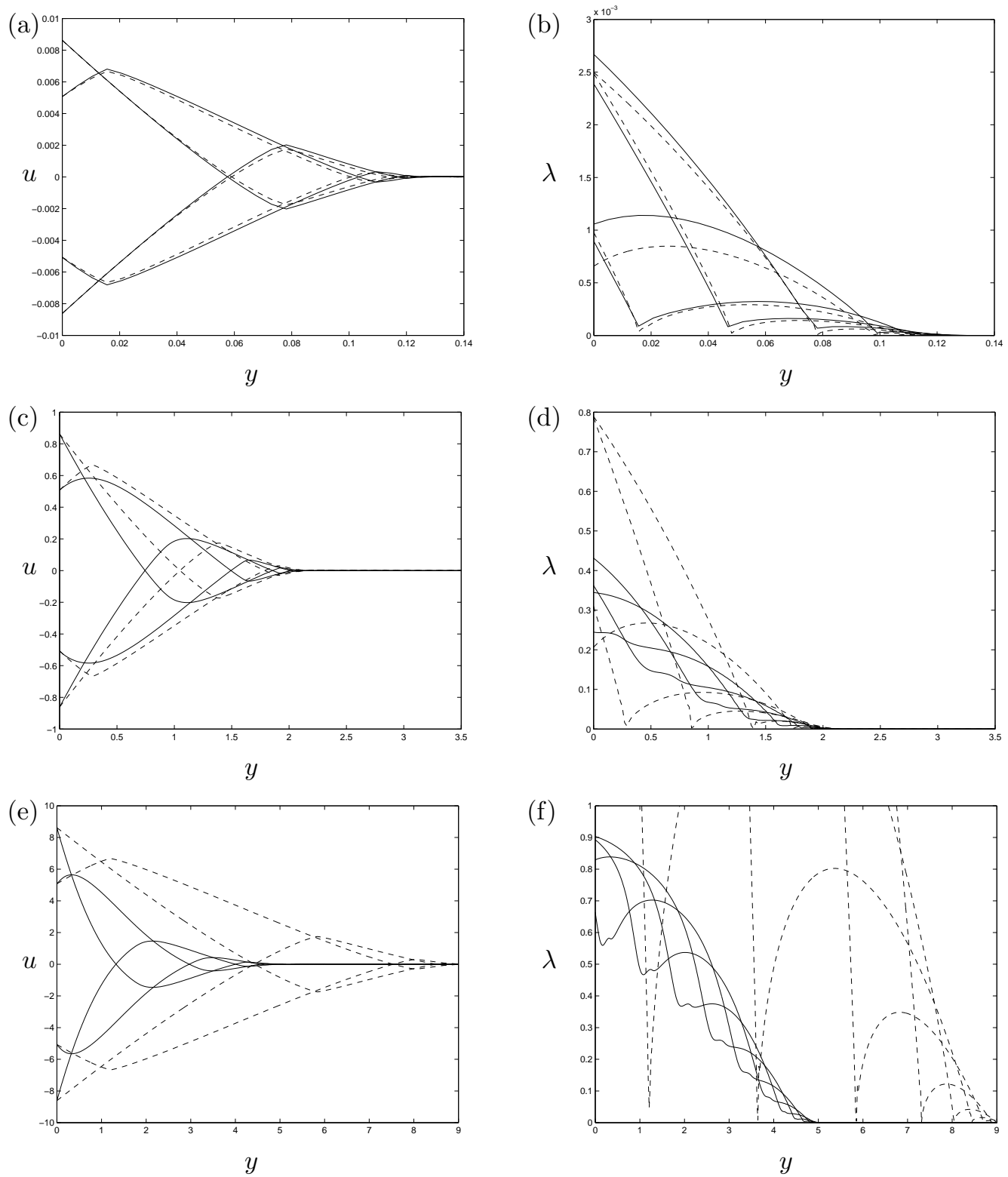

Figure 10: Antithixotropic regime A1 $(\nu>0)$ : snapshots of the numerical solutions (solid lines) and fast-adjusting asymptotic solutions (dashed lines) with $a=1, b=3 / 10, c=2$, $d=1, \omega=1$ and $(\mathrm{a}, \mathrm{b}) \epsilon=0.01 ;(\mathrm{c}, \mathrm{d}) \epsilon=1 ;(\mathrm{e}, \mathrm{f}) \epsilon=10$. Plots (a), (c) and (e) show the velocity $u(y, t)$ at $t=100+n \pi / 2$ for $n=0,1,2$ and 3 ; and plots (b), (d) and (f) show the structure parameter $\lambda(y, t)$ at $t=100+n \pi / 4$ for $n=0,1,2,3$. 

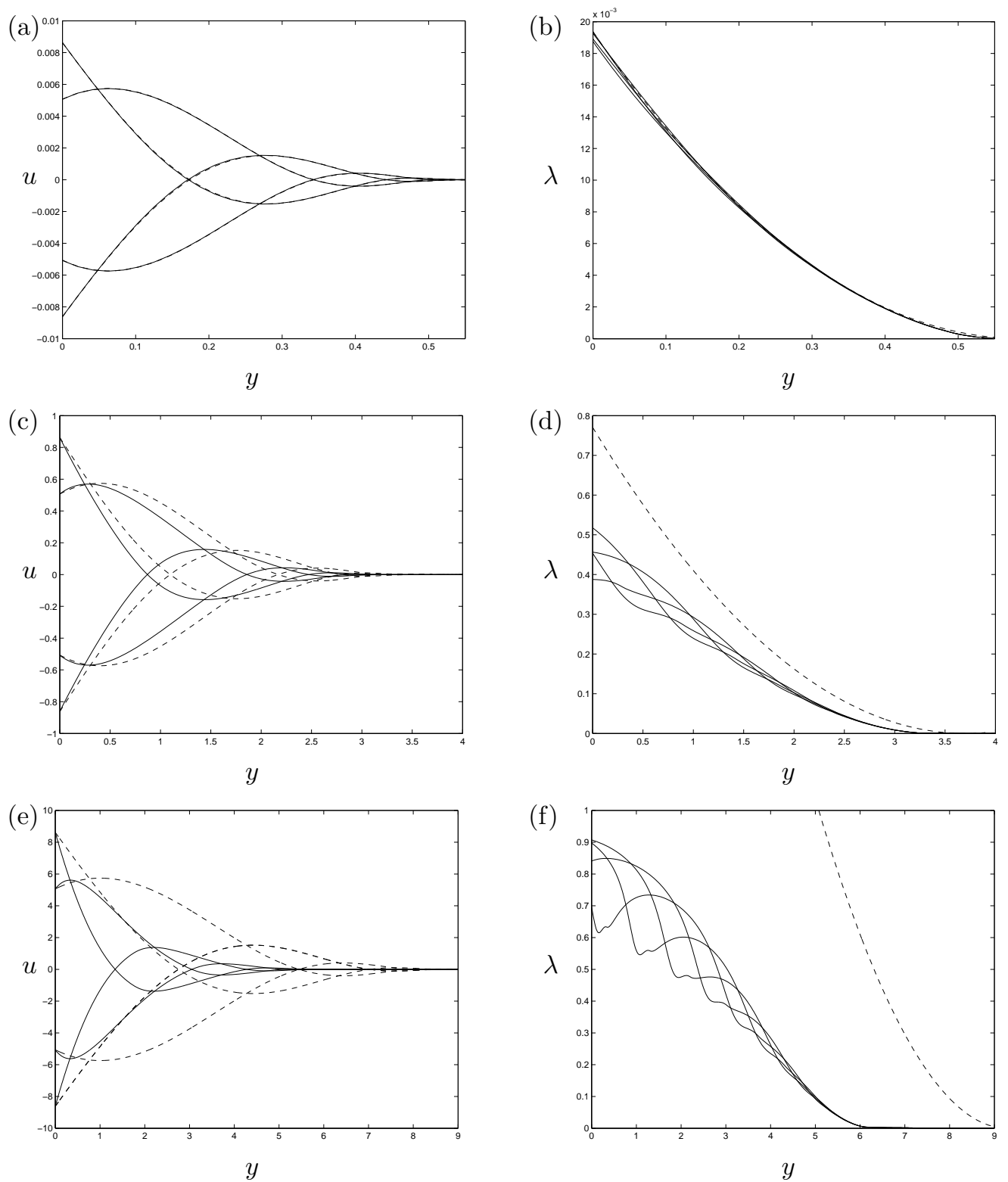

Figure 11: Antithixotropic regime A2 $(\nu<0)$ : snapshots of the numerical solutions (solid lines) and slow-adjusting asymptotic solutions (dashed lines) with $a=1, b=3 / 4, c=2$, $d=1, \omega=1$ and (a, b) $\epsilon=0.01 ;(\mathrm{c}, \mathrm{d}) \epsilon=1 ;(\mathrm{e}, \mathrm{f}) \epsilon=10$. Plots (a), (c) and (e) show the velocity $u(y, t)$ at $t=100+n \pi / 2$ for $n=0,1,2$ and 3 ; and plots (b), (d) and (f) show the structure parameter $\lambda(y, t)$ at $t=100+n \pi / 4$ for $n=0,1,2,3$. 

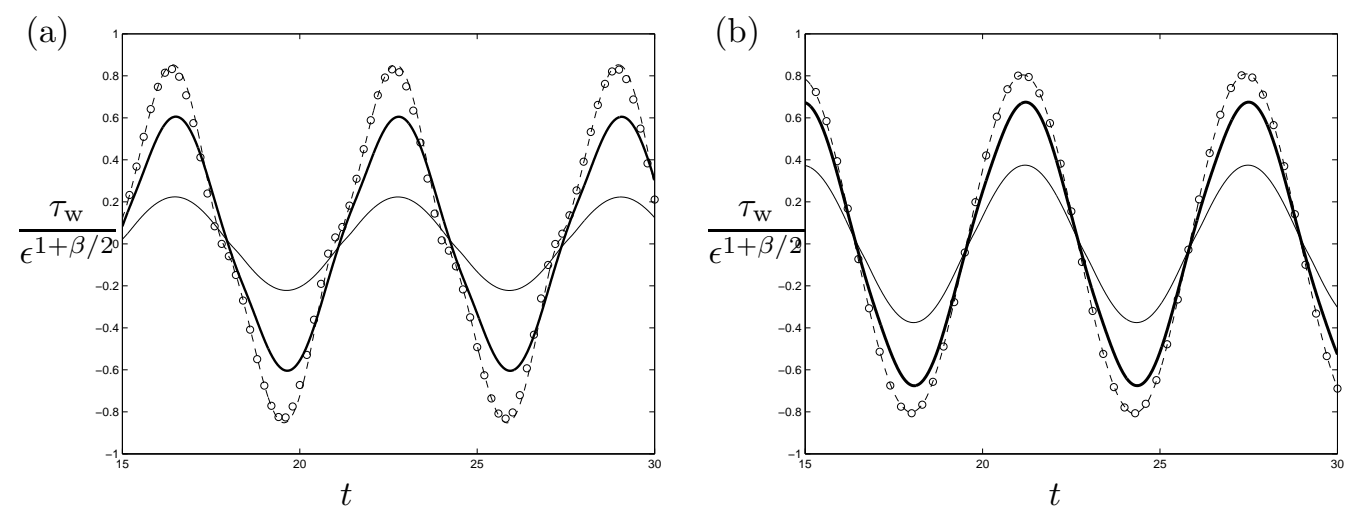

Figure 12: Normalised shear stress at the wall, $\tau_{\mathrm{w}}(t) / \epsilon^{1+\beta / 2}$, with $a=1, c=2, d=1$ and $\omega=1$ and (a) $b=3 / 10$, (b) $b=3 / 4$. Figure (a) is the shear stress for regime A1 $(\nu>0)$ and figure (b) is the shear stress for regime A2 $(\nu<0)$. The circles represent the asymptotic solution; lines represent the numerical solutions for $\epsilon=0.01$ (dashed); $\epsilon=1$ (thick solid); $\epsilon=10$ (thin solid). 


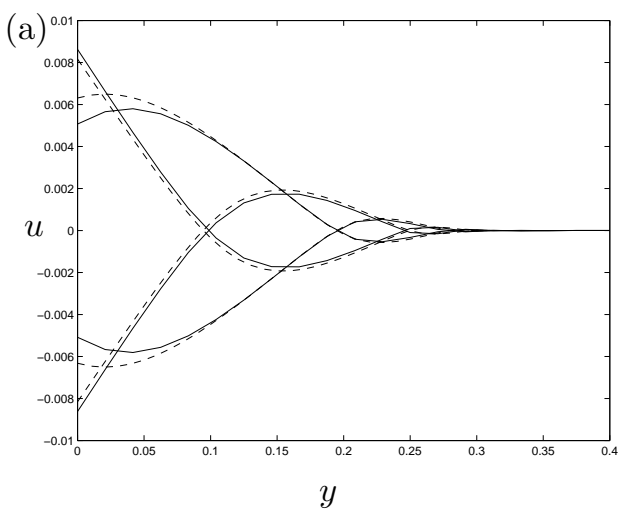

(b)

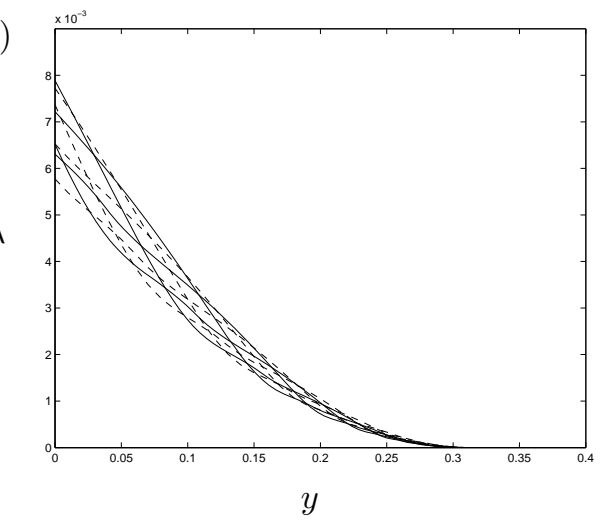

(c)

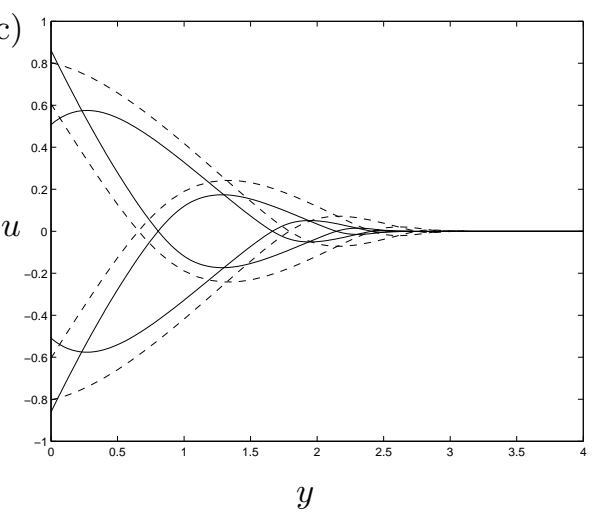

(d)

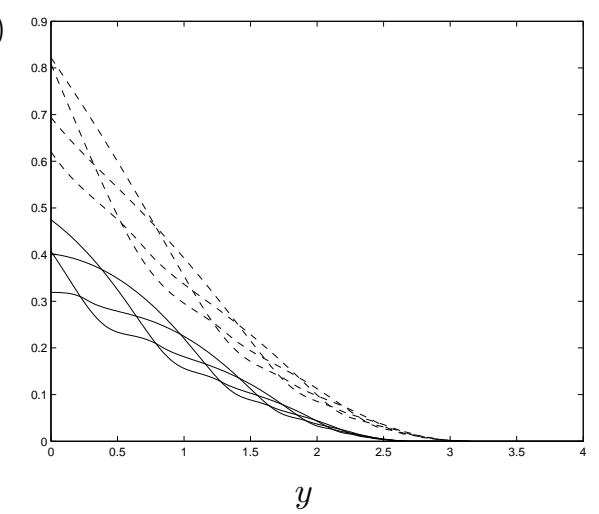

(e)

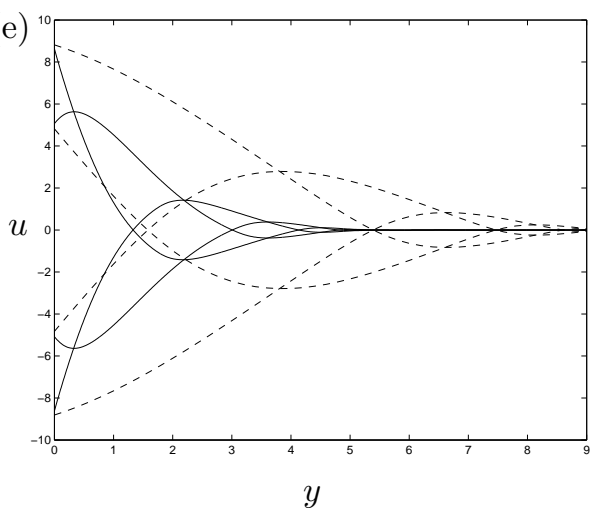

(f)

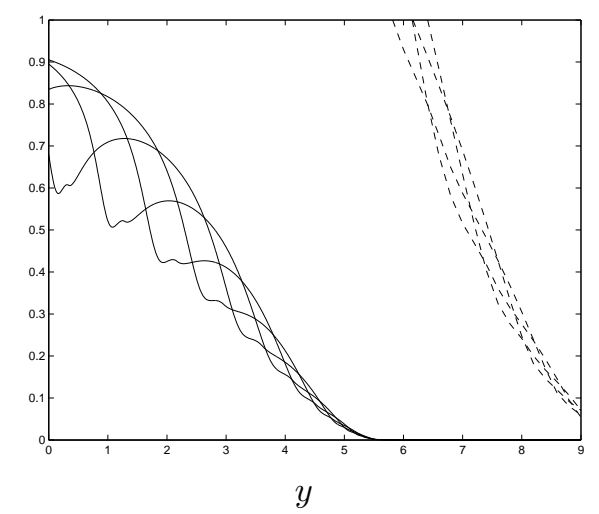

Figure 13: Antithixotropic marginal regime $(\nu=0)$ : snapshots of the numerical solutions (solid lines) and marginal asymptotic solutions (dashed lines) with $a=1, b=1 / 2, c=2$, $d=1, \omega=1$ and (a, b) $\epsilon=0.01$; (c, d) $\epsilon=1 ;(\mathrm{e}, \mathrm{f}) \epsilon=10$. Plots (a), (c) and (e) show the velocity $u(y, t)$ at $t=100+n \pi / 2$ for $n=0,1,2$ and 3 ; and plots (b), (d) and (f) show the structure parameter $\lambda(y, t)$ at $t=100+n \pi / 4$ for $n=0,1,2,3$. 


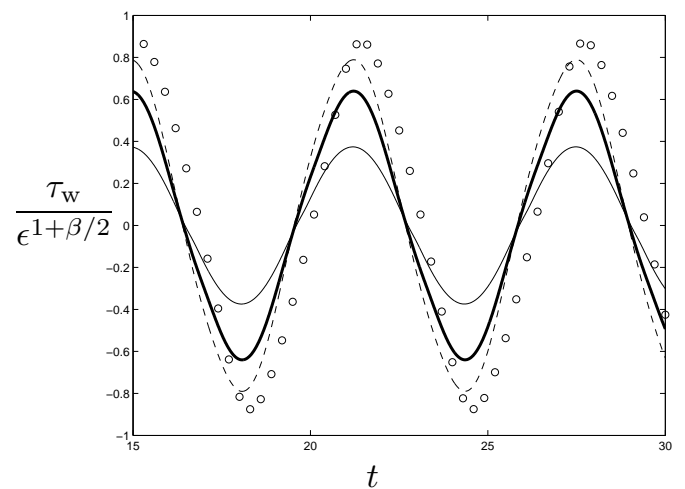

Figure 14: Antithixotropic marginal regime: normalised shear stress at the wall, $\tau_{\mathrm{w}}(t) / \epsilon^{1+\beta / 2}$, with $a=1, b=1 / 2, c=2, d=1$ and $\omega=1$. The circles represent the asymptotic solution; lines represent the numerical solutions for $\epsilon=0.01$ (dashed); $\epsilon=1$ (thick solid); $\epsilon=10$ (thin solid). 
(a)

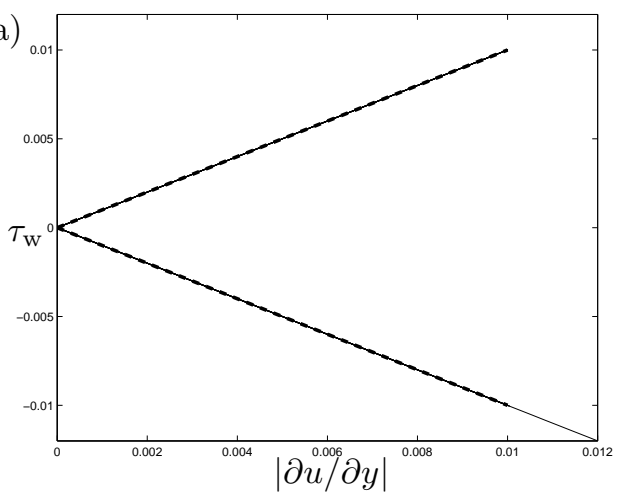

(c)

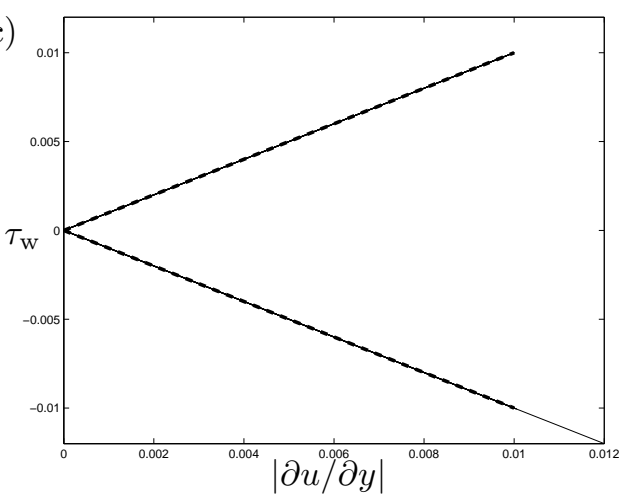

(e)

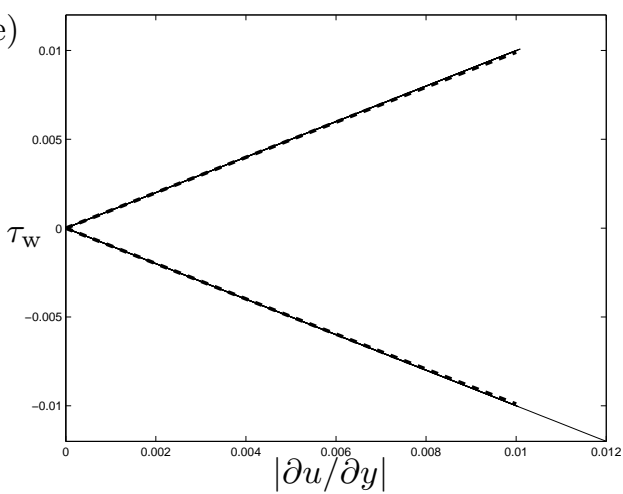

(b)

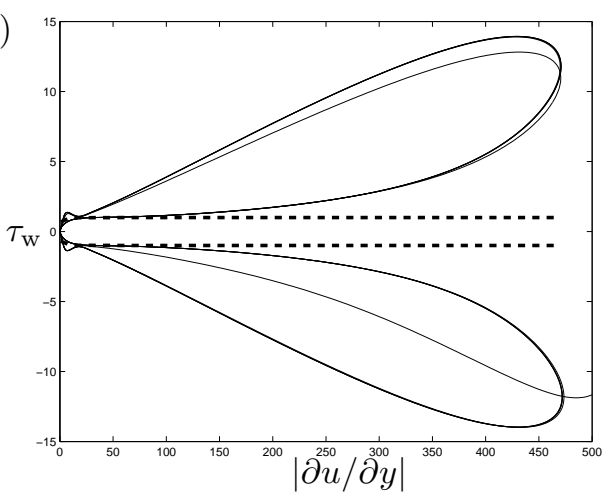

(d)

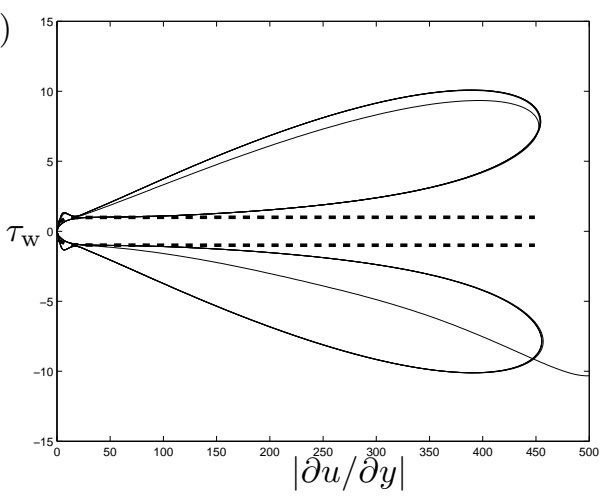

(f)

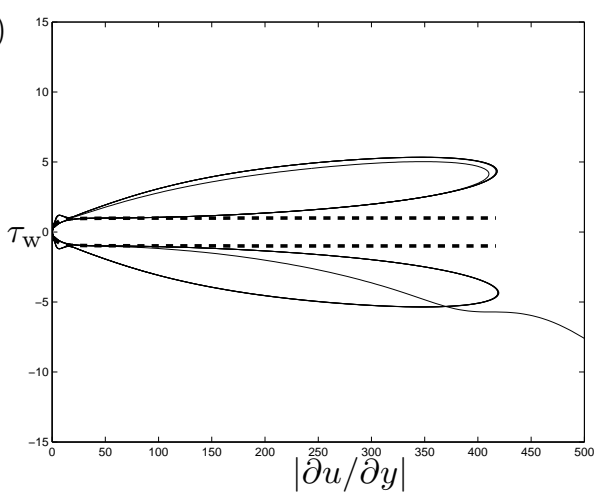

Figure 15: Rheograms for thixotropic fluids with $a=2, b=1, c=1, \omega=1$ and (a, b) $d=1 / 3 ;(\mathrm{c}, \mathrm{d}) d=1 / 2 ;(\mathrm{e}, \mathrm{f}) d=1$. Plots (a), (c) and (e) show the numerical results for $\epsilon=0.01$ (solid lines) and plots (b), (d) and (f) show the numerical results for $\epsilon=10$ (solid lines); dashed lines in each case represent the instantaneous equilibrium shear stress $\tau_{\text {eq }}$. 


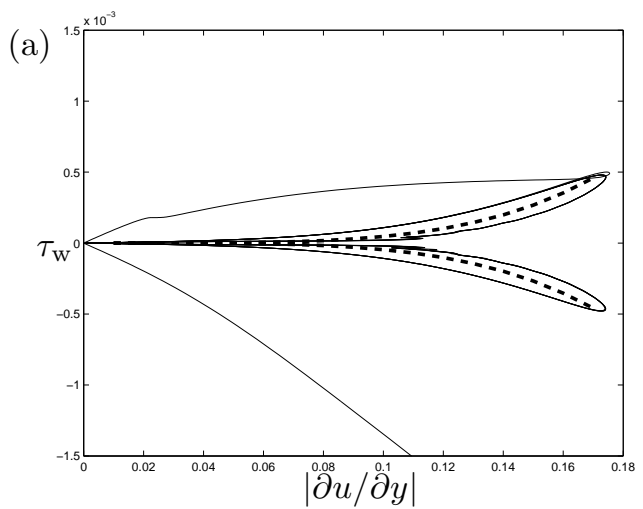

(b)

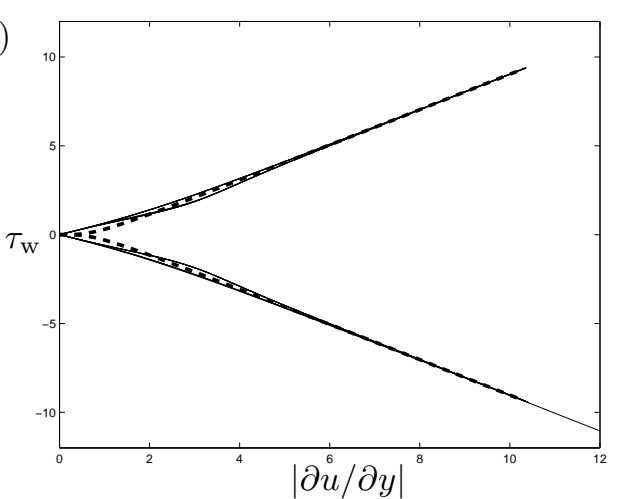

(c)

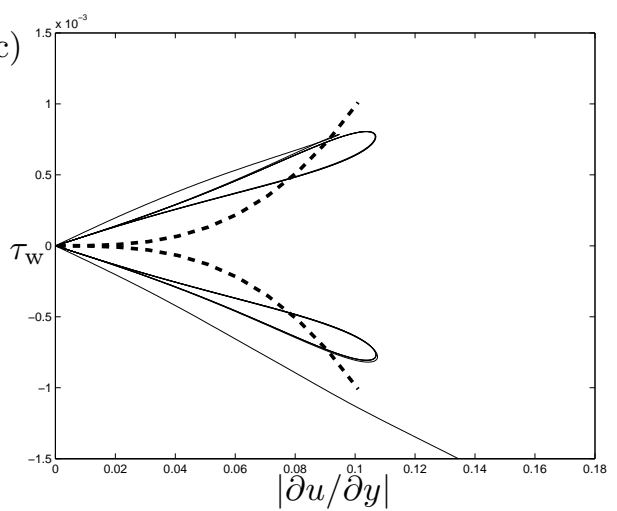

(d)

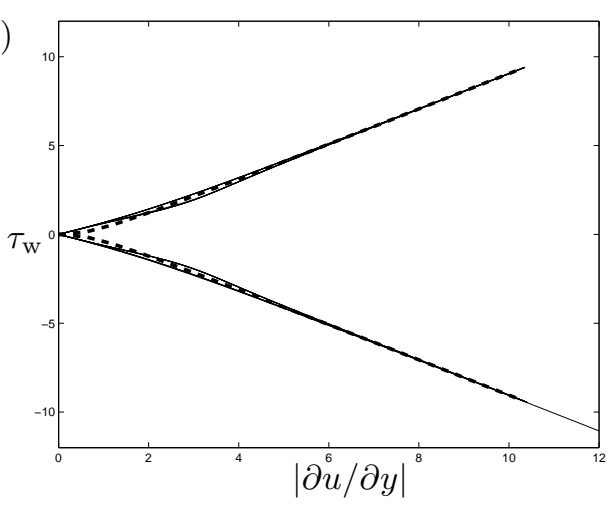

(e)

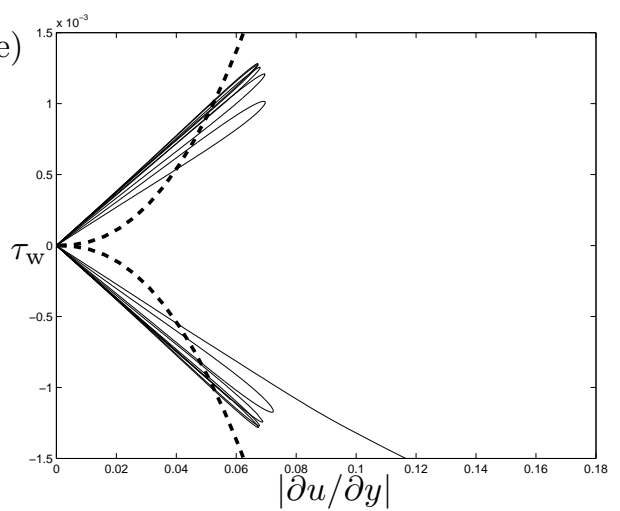

(f)

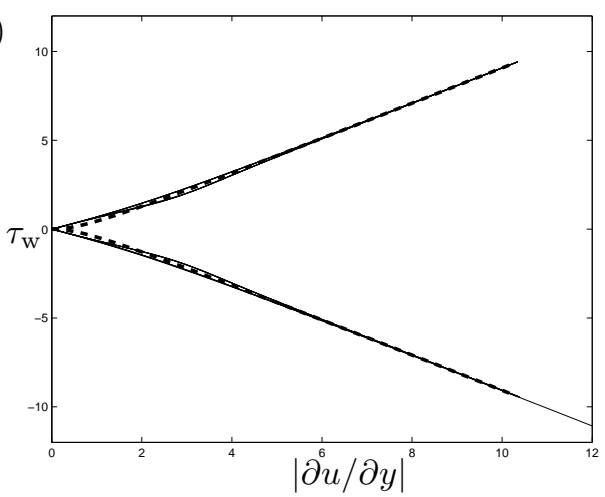

Figure 16: Rheograms for antithixotropic fluids with $a=1, c=2, d=1, \omega=1$ and (a, b) $b=3 / 10 ;(\mathrm{c}, \mathrm{d}) b=1 / 2 ;(\mathrm{e}, \mathrm{f}) b=3 / 4$. Plots (a), (c) and (e) show the numerical results for $\epsilon=0.01$ (solid lines) and plots (b), (d) and (f) show the numerical results for $\epsilon=10$ (solid lines); dashed lines in each case represent the instantaneous equilibrium shear stress $\tau_{\text {eq. }}$ 


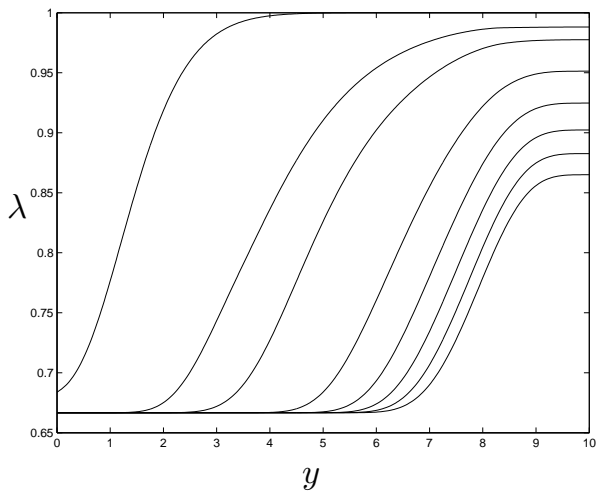

Figure 17: 'Pseudo-Newtonian' regime: snapshots of the structure parameter $\lambda(y, t)$ at (from left to right) $t=1,10,25,100,200,300,400,500$, with $a=1, b=1, c=1, d=1, \epsilon=1$ and $\kappa_{2}=2$. 



\section{RECENT REPORTS}

12/31 Convergent evolution of spiny mollusk shells points to elastic energy minimum

Chirat

Moulton

Shipman

Goriely

12/32 Three-dimensional oblique water-entry problems at small deadrise angles

Moore

Howison

Ockendon

Oliver

12/33 Second weak order explicit stabilized methods for stiff stochastic differential equations

Abdulle

Vilmart

Zygalakis

12/34 The sensitivity of Graphene 'Snap-through' to substrate geometry Wagner

Vella

12/35 The physics of frost heave and ice-lens growth

Peppin

Style

12/36 Finite Element Simulation of Dynamic Wetting Flows as an Inter-

Sprittles face Formation Process

Shikhmurzaev

12/37 The Dynamics of Liquid Drops and their Interaction with Solids of Varying Wettabilities

Sprittles

Shikhmurzaev

12/38 Dispersal and noise: Various modes of synchrony in ecological oscillators

Bressloff

Lai

12/39 Boundary conditions for free surface inlet and outlet problems

Taroni

Breward

Howell

Oliver

12/40 A Branch and Bound Algorithm for the Global Optimization of Hessian Lipschitz Continuous Functions

Fowkes

Gould

Farmer

12/41 The Orthogonal Gradients Method: a Radial Basis Functions

Piret Method for Solving Partial Differential Equations on Arbitrary Surfaces

12/42 Squeeze-Film Flow in the Presence of a Thin Porous Bed, with Application to the Human Knee Joint

Knox

Wilson

Duffy

McKee

12/43 Gravity-driven draining of a thin rivulet with constant width down

Paterson a slowly varying substrate

Wilson

Duffy

12/44 The 'Sticky Elastica': Delamination blisters beyond small deformations 
12/45 Stochastic models of intracellular transport

Bressloff

Newby

12/46 The effects of noise on binocular rivalry waves: a stochastic neu-

Webber ral field model

Bressloff

12/47 An Ensemble Bayesian Filter for State Estimation

Farmer

12/48 Simulation of cell movement through evolving environment: a fictitious domain approach

Séguis

Burrage

Erban

Kay

12/49 The Mathematics of Liquid Crystals: Analysis, Computation and

Majumdar Applications

12/50 Fourier spectral methods for fractional-in-space reaction-diffusion equations

Bueno-Orovio

Kay

Burrage

12/51 Meniscal tear film fluid dynamics near Marx's line

Zubkov

Breward

Gaffney

12/52 Validity of the Cauchy-Born rule applied to discrete cellular-scale

Davit models of biological tissues

Osborne

Byrne

Gavaghan

Pitt-Francis

12/53 A thin rivulet or ridge subject to a uniform transverse shear stress

Sullivan at its free surface due to an external airflow

Paterson

Wilson

Duffy

Copies of these, and any other OCCAM reports can be obtained from:

Oxford Centre for Collaborative Applied Mathematics

Mathematical Institute

24 - 29 St Giles'

Oxford

OX1 3LB

England

www.maths.ox.ac.uk/occam 